\title{
Massive star-formation in G24.78+0.08 explored through VLBI maser observations ${ }^{\star}$
}

\author{
L. Moscadelli ${ }^{1}$, C. Goddi ${ }^{2}$, R. Cesaroni ${ }^{1}$, M. T. Beltrán ${ }^{3}$, and R. S. Furuya ${ }^{4}$ \\ 1 INAF - Osservatorio Astrofisico di Arcetri, Largo E. Fermi 5, 50125 Firenze, Italy \\ e-mail: mosca@arcetri.astro.it \\ 2 Harvard-Smithsonian Center for Astrophysics, 60 Garden Street, Cambridge, MA 02138, USA \\ 3 Departament d'Astronomia i Meteorologia, Universitat de Barcelona, Av. Diagonal 647, 08028, Barcelona, Catalunya, Spain \\ ${ }^{4}$ Subaru Telescope, National Astronomical Observatory of Japan, 650 North A'ohoku Place, Hilo, HI 96720, USA
}

Received 9 May 2007 / Accepted 10 July 2007

\section{ABSTRACT}

\begin{abstract}
Context. Previous interferometric observations have demonstrated that, across a distance of a few $0.1 \mathrm{pc}$, the high-mass star forming region (SFR) G24.78+0.08 contains at least four distinct centers of massive star formation, possibly in different evolutionary stages. Aims. This study aims to provide a detailed picture of the physical environment and the gas kinematics in a cluster of high-mass YSOs.

Methods. Using EVN (single epoch) and VLBA (four epochs) phase-referenced observations, we have derived the absolute positions and velocities for $6.7 \mathrm{GHz}$ methanol and $22.2 \mathrm{GHz}$ water masers, respectively. Using the BIMA and VLA interferometers, positions and line of sight velocities of $95 \mathrm{GHz}$ and $44 \mathrm{GHz}$ methanol masers, are also obtained. The derived interferometric and VLBI maser maps are compared with previous sub-arcsecond maps of the $\mathrm{G} 24.78+0.08$ region, observed in thermal continuum and molecular line tracers.

Results. In the hot molecular cores G24 A1 and G24 A2, $6.7 \mathrm{GHz}$ methanol and $22.2 \mathrm{GHz}$ water masers are emerging at similar positions and line of sight velocities, which suggests that in both cores a same YSO is responsible for the excitation of the two types of maser emission. At the center of the G24 A1 core, water masers distribute along an arc at the border of a hyper-compact HII region (of size $\approx 1000 \mathrm{AU}$ ) and expand away from the center of the HII region with high velocities $\left(\approx 40 \mathrm{~km} \mathrm{~s}^{-1}\right)$. We think that such a fast expansion is driven by a strong stellar wind emitted by the star exciting the hyper-compact HII region. This outflowing motion might dominate the gas kinematics of G24 A1 also at larger ( $\approx 0.1 \mathrm{pc})$ scale, where a well defined velocity gradient in the $\mathrm{CH}_{3} \mathrm{CN}_{\text {line and }}$ $6.7 \mathrm{GHz}$ masers is observed. Alternatively, water masers might mark the border of the dynamical interaction between the evolving star and harboring core, and the gas surrounding the hyper-compact HII region, not yet affected by the expansion of the ionized gas, might be still rotating and, perhaps, slightly contracting. In the G24 C core, water maser spots show very fast $\left(100-200 \mathrm{~km} \mathrm{~s}^{-1}\right)$ and nearly parallel proper motions, which might indicate that the water maser emission is tracing a collimated jet.
\end{abstract}

Key words. masers - stars: formation - ISM: kinematics and dynamics

\section{Introduction}

Understanding the process of high-mass star formation represents a challenge from both a theoretical and observational point of view. By simply scaling the theory of star formation from lowto high-mass stars, one finds that the intense radiation pressure from the star luminosity may be sufficient to reverse the accretion flow and prevent matter from reaching the star. The "standard" theory predicts that this occurs for stars having masses in excess of $8 M_{\odot}$ (Palla \& Stahler 1993), leading to the paradoxical conclusion that stars above this limit should not exist. In order to circumvent this dilemma, it has been proposed that highmass stars might build up via merging of lower mass $\left(<8 M_{\odot}\right)$ objects (coalescence model - Bonnell \& Bate 2002), or, alternatively, by accreting material from the infalling envelope via a disk, as it happens in low-mass stars (accretion model - Yorke \& Sonnhalter 2002). In the last few years, a sample of highmass Young Stellar Objects (YSOs) in the earliest evolutionary stages has been investigated, inferring evidence of rotational (see Cesaroni 2005, for a review) and infalling motions (e.g.,

* Tables 1, 5, 6 and Appendices A-C are only available in electronic form at http://www . aanda. org
Beltrán et al. 2006). Notwithstanding this evidence, it remains to be established whether the rotating and infalling material will eventually accrete onto the star. This result would strongly support the accretion scenario. However, the available observational evidence is still inadequate to discern between different alternative scenarios as it gives contradictory results. On the one hand, thermal transitions of molecules like $\mathrm{CH}_{3} \mathrm{CN}$ and $\mathrm{NH}_{3}$ trace infall/rotation on scales of $\sim 0.01$ pc (Beltrán et al. 2004; Beltrán et al. 2006). On the other hand, $\mathrm{H}_{2} \mathrm{O}$ masers clearly trace expansion on much smaller scales ( $\sim 0.001 \mathrm{pc}$ ) (Goddi et al. 2005; Moscadelli et al. 2005). Thus, it is necessary to trace the infalling gas in the close proximity $(<0.001 \mathrm{pc})$ of the star, in order to definitely clarify whether it accretes onto the star or not. This is not trivial from an observational point of view, since high-mass stars are on average more distant from us (typically $\geq 1 \mathrm{kpc}$ ) and form fast and ignite whilst still enshrouded in dense dusty envelopes, opaque to optical and, often, near-infrared wavelengths.

The above mentioned observational limitations can be bypassed by Very Long Baseline Interferometry (VLBI) observations of molecular masers, which may easily achieve angular resolutions of $\sim 1-10$ mas, and allow accurate measurements of positions and 3-dimensional (3D) velocities of the maser spots, 
thus investigating the gas kinematics around YSOs in great detail. Several species of molecular masers $\left(\mathrm{OH}, \mathrm{CH}_{3} \mathrm{OH}, \mathrm{H}_{2} \mathrm{O}\right)$ are commonly observed towards high-mass SFRs, but it is not clear how the different types of masers are related to each other. The small number $(<10)$ of maser associations observed up to now with VLBI, suggests that different maser types trace distinct environments. VLBI multi-epoch observations have clarified that water masers originate from shocks associated with winds and/or jets ejected by the massive YSOs (e.g. Torrelles et al. 2003; Goddi et al. 2005; Moscadelli et al. 2005), whereas $1.6 \mathrm{GHz} \mathrm{OH}$ masers seem to arise from rotating disks about protostars (e.g. Edris et al. 2005). Instead, the birthplace of $6.7 \mathrm{GHz}$ methanol masers is still more uncertain. High-resolution imaging has shown that some $\mathrm{CH}_{3} \mathrm{OH}$ sources have maser spots in linear or arc-like structures with velocity gradients compatible with Keplerian rotation, suggesting that these masers may originate in the innermost portions of a disk rotating about the protostar (Norris et al. 1998; Edris et al. 2005). In other cases, the $6.7 \mathrm{GHz}$ spots are distributed parallel to the axis of the jet/outflow as traced by the shocked $\mathrm{H}_{2}$ emission at $2.12 \mu \mathrm{m}$ and it has been proposed that the methanol maser emission may trace the shock at the interface between the jet and the circumstellar molecular gas (De Buizer 2003).

For a thorough analysis of the environment of newly formed massive (proto-)stars, the results of VLBI maser observations need to be complemented with interferometric radio and infrared observations of molecular thermal lines of the larger scale environment. The aim of the present paper is a detailed (VLBI) study of various molecular maser species in the SFR G24.78+0.08, towards which previous (IR, $\mathrm{mm}$, and radio) interferometric observations have provided a very detailed description of the circumstellar gas on scales $\sim 0.01-0.1$ pc (Codella et al. 1997; Furuya et al. 2002; Cesaroni et al. 2003; Beltrán et al. 2004; Beltrán et al. 2005). The SFR is located at a distance of $7.7 \mathrm{kpc}$ and has a large bolometric luminosity $\left(\leq 7 \times 10^{4} L_{\odot}\right)$. Furuya et al. (2002) firstly detected three molecular cores (in the $2 \mathrm{~mm}$ continuum emission) and two HII regions (one of these embedded in one of the cores) spread over $\sim 0.4 \mathrm{pc}$. The region contains at least four distinct centers of star formation, named A, B, C, and D, which Furuya et al. (2002) speculate to be on different evolutionary states. The molecular core A and the source B have associated a HII region (Codella et al. 1997) and, hence, are already ZAMS stars, whereas the other two appear to be less evolved. Hence, the G24.78+0.08 SFR provides the rare opportunity to study a cluster of high-mass YSOs in different evolutionary stages. Both cores $\mathrm{A}$ and $\mathrm{C}$ power a bipolar molecular outflow (Furuya et al. 2002).

$1.4 \mathrm{~mm}$ Plateau de Bure Interferometer (PdBI) 1-arcsec angular resolution observations by Beltrán et al. (2004) have resolved the structure of the G24 A core into two distinct subcores: G24 A1, the one towards southeast (SE), and G24 A2, towards northwest (NW). G24 A1 is associated with the most compact HII region, while G24 A2 presents strong pointlike emission from 3.6 to $8 \mu \mathrm{m}$ (GLIMPSE) but weak radio $22 \mathrm{GHz}$ continuum emission. Both G24 A1 and A2 are hot molecular cores (HMCs) (Beltrán et al. 2004), with a $V_{\text {LSR }}$ gradient along a northeast-southwest (NE-SW) direction, approximately perpendicular to the axis of the ${ }^{12} \mathrm{CO}$ molecular outflow. This fact suggests that the two cores might be rotating around the outflow axis (Beltrán et al. 2004). Towards G24 A1 and A2 several species of molecular masers have been detected: $6.7 \mathrm{GHz} \mathrm{CH}_{3} \mathrm{OH}$, observed with the Australian Compact Array (ATCA) by Walsh

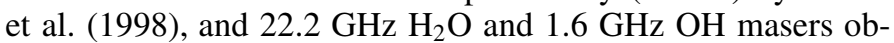
served with the VLA by Forster \& Caswell (1989). With the angular resolution of a few tenths of arcsecond provided by these interferometers, the maser spots of the three maser species exhibit an elongated spatial distribution, approximately parallel to the axis of the $\mathrm{CO}$ molecular outflow. Unlike the two HMCs A1 and $\mathrm{A} 2$, core $\mathrm{C}$ has a relatively low temperature $(\sim 30 \mathrm{~K})$ and no radio continuum emission. Also in this case, evidence for rotation about the outflow axis is found (Beltrán et al. 2004). Both $\mathrm{H}_{2} \mathrm{O}$ and Class $\mathrm{I} \mathrm{CH}_{3} \mathrm{OH}$ masers are also associated with core $\mathrm{C}$.

In Sect. 2, we describe the observations of the Class I $95 \mathrm{GHz}$ $\mathrm{CH}_{3} \mathrm{OH}$ masers with the BIMA interferometer, $44 \mathrm{GHz} \mathrm{CH}_{3} \mathrm{OH}$ and $22 \mathrm{GHz} \mathrm{H}_{2} \mathrm{O}$ masers with the Very Large Array (VLA), $6.7 \mathrm{GHz} \mathrm{CH}_{3} \mathrm{OH}$ (one epoch) masers with the European VLBI Network (EVN) and 1.6 GHz OH (one epoch) and $22.2 \mathrm{GHz}$ $\mathrm{H}_{2} \mathrm{O}$ masers (four epochs) with the Very Long Baseline Array (VLBA). Section 3 reports the detection of rich clusters of molecular masers towards cores A1, A2, and C, and describes the remarkable linear and arcuate structures of methanol and water maser spots discovered in correspondence of cores A1 and C. In Sect. 4 we discuss the association of $6.7 \mathrm{GHz}$ methanol and $22.2 \mathrm{GHz}$ water masers. Sections 5 and 6 consider possible scenarios to explain the origin of the observed maser structures, and the main conclusions of our study are reported in Sect. 7.

\section{Observations and data reduction}

\subsection{BIMA observations}

Observations of the $9_{2}-10_{1} \mathrm{~A}^{+}\left(v_{0}=95.2 \mathrm{GHz}\right)$ transition of $\mathrm{CH}_{3} \mathrm{OH}$ were conducted with the 10 -antenna BIMA ${ }^{1}$ array in the A configuration on 2003 January 08 and 18. The digital correlator was configured to observe simultaneously the continuum emission and some molecular lines. The phase center was located at $\alpha(\mathrm{J} 2000)=18^{\mathrm{h}} 36^{\mathrm{m}} 12^{\mathrm{s}} .66, \delta(\mathrm{J} 2000)=$ $-7^{\circ} 12^{\prime} 10^{\prime}{ }^{\prime} 15$. The spectral resolution of the observations was $0.31 \mathrm{~km} \mathrm{~s}^{-1}$ for $\mathrm{CH}_{3} \mathrm{OH}$ at $95.169438 \mathrm{GHz}$ and the bandwidth was $25 \mathrm{MHz}$. Bandpass calibration was achieved by observing the quasar 3C 345 . Amplitude and phase were calibrated by observations of the quasar 1743-038, whose flux density was determined relative to Mars. The uncertainty in the amplitude calibration is estimated to be $\sim 20 \%$. The data were calibrated using standard procedures in the MIRIAD software package (Sault et al. 1995). We subtracted the continuum from the line emission directly in the $(u, v)$ domain. The maps were done by using natural weighting, with a resulting synthesized beam of $0.75 \times$ $0.58, \mathrm{PA}=31^{\circ}$. The $1-\sigma$ noise in the single channel map was $70 \mathrm{mJy}^{\text {beam }}{ }^{-1}$.

\subsection{VLA observations}

\subsubsection{Class I $\mathrm{CH}_{3} \mathrm{OH} 44 \mathrm{GHz}$ masers}

Observations of the $7_{0}-6_{1} \mathrm{~A}^{+}\left(v_{0}=44.069430 \mathrm{GHz}\right)$ transition of $\mathrm{CH}_{3} \mathrm{OH}$ were conducted on 2005 August 23 using the Very Large Array (VLA) ${ }^{2}$ in its C configuration, for a total observing time of $4.5 \mathrm{~h}$. The phase center of the VLA (44 GHz and $22.2 \mathrm{GHz}$ ) observations was the same as for the BIMA observations.

The observations were carried out using a total bandwidth of $3.125 \mathrm{MHz}$ with 127 spectral channels, providing a total velocity coverage of $21.3 \mathrm{~km} \mathrm{~s}^{-1}$ and a velocity resolution of $0.17 \mathrm{~km} \mathrm{~s}^{-1}$.

\footnotetext{
1 The BIMA array was operated by the Berkeley-Illinois-Maryland Association with support from the National Science Foundation.

2 The VLA is operated by the National Radio Astronomy Observatory (NRAO).
} 
The observations were performed using the "fast switching" method, where 4 min scans of G24.78+0.08 were alternated with 1 min scans of the nearby phase calibrator 1832-105. Referenced pointing was performed approximately once per hour. Absolute amplitude calibration was obtained from observations of the quasar $3 \mathrm{C} 286$, with a flux density of $\approx 1 \mathrm{Jy}$. The error in the absolute flux density calibration is estimated to be within $30 \%$ in the $7 \mathrm{~mm}$ band.

The VLA data were edited, calibrated, and imaged using the NRAO's Astronomical Image Processing System (AIPS) package. In order to increase the signal-to-noise ratio $(S / N)$, a strong, spectrally unblended maser component (at $115.2 \mathrm{~km} \mathrm{~s}^{-1}$ ) was identified and the corresponding channel was self-calibrated in phase and amplitude; this calibration was then applied to the other channels. After self-calibration, the rms noise of the reference channel decreased by about one order of magnitude, going from 0.15 to $0.02 \mathrm{Jy}$ beam $^{-1}$. We produced (naturally weighted) maps extended over an area of $50^{\prime \prime} \times 50^{\prime \prime}$ and covering the velocity range from 105.8 to $122.4 \mathrm{~km} \mathrm{~s}^{-1}$. The CLEAN beam was an elliptical Gaussian with a $F W H M$ size of $0.53 \times 0.45$, $\mathrm{PA}=178^{\circ}$. Each channel map was searched for emission above a conservative detection threshold taken equal to the absolute value of the minimum in the map (varying across the channels in the range $5-7 \sigma$, where $\sigma \approx 0.01-0.04 \mathrm{Jy} \mathrm{beam}^{-1}$ ). The detected maser spots were fitted with two-dimensional elliptical Gaussians, determining position, flux density, and $F W H M$ size of the emission. Spectrally and spatially (within the FWHM beam size) contiguous maser spots were considered as single "features". The most intense features were found to consist of up to $20-30$ spots.

\subsection{2. $\mathrm{H}_{2} \mathrm{O} 22.2 \mathrm{GHz}$ masers}

Observations of the $6_{16}-5_{23} \mathrm{H}_{2} \mathrm{O}$ maser line (rest frequency $22235.080 \mathrm{MHz}$ ) were conducted on 2003 September 17 using the VLA in its A configuration, for a total observing time of $20 \mathrm{~min}$.

The observations were carried out using a total bandwidth of $3.125 \mathrm{MHz}$ with 64 spectral channels, providing a total velocity coverage of $42 \mathrm{~km} \mathrm{~s}^{-1}$ and a velocity resolution of $0.66 \mathrm{~km} \mathrm{~s}^{-1}$. Employing the "fast switching" observing mode, 10 min scan of G24.78+0.08 were alternated with 2 min scans of the nearby phase calibrator $1851+005$. Absolute amplitude calibration was obtained from observations of the quasar 3C 286, with a flux density of $\approx 2.5 \mathrm{Jy}$.

After applying the self-calibration corrections derived from a strong, spectrally isolated channel (at $112.3 \mathrm{~km} \mathrm{~s}^{-1}$ ), we produced (naturally weighted) maps extended over an area of $20^{\prime \prime} \times$ $20^{\prime \prime}$ and covering the velocity range from 90.6 to $131.4 \mathrm{~km} \mathrm{~s}^{-1}$. The CLEAN beam was an elliptical Gaussian with a $F W H M$ size of $0{ }^{\prime} .29 \times 00^{\prime} 17, \mathrm{PA}=93^{\circ}$. Each channel map was searched for emission above the absolute value of the minimum in the map (varying across the channels in the range 5-7 $\sigma$, where $\sigma \approx 0.007-0.31 \mathrm{Jy} \mathrm{beam}^{-1}$ ). The detected maser spots were fitted with two-dimensional elliptical Gaussians, determining position, flux density, and $F W H M$ size of the emission.

\subsection{EVN observations}

$\mathrm{G} 24.78+0.08$ was observed in the $5_{1}-6_{0} \mathrm{~A}^{+}$line of methanol at $6.668 \mathrm{GHz}$ using the European VLBI Network (EVN) in two separate 6-h runs on June 5 and 6, 2003. The antennae involved in the observations were Cambridge, Jodrell, Onsala,
Effelsberg, Hartebeesthoek, and Torun. The observations were performed in phase-reference mode, alternating scans on the maser source, G24.78+0.08, and the nearby (2:3 apart) VLBA calibrator J1825-0737, with a switching cycle of $5.5 \mathrm{~min}$. For the purpose of bandpass and phase calibration, 5-min scans on several continuum sources $(\mathrm{J} 1751+09$, J1642+39, J2101+03, $\mathrm{J} 2253+16)$ were observed every $1.5 \mathrm{~h}$. The total on-source integration time for the maser target was about $5 \mathrm{~h}$. Dual circular polarization was recorded with a $8 \mathrm{MHz}$ bandwidth centered at the LSR velocity of $80 \mathrm{~km} \mathrm{~s}^{-1}$. The data were processed with the MKIV correlator at the Joint Institute for VLBI in Europe (JIVE - Dwingeloo, The Netherlands), obtaining 1024 spectral channels with a separation of $0.35 \mathrm{~km} \mathrm{~s}^{-1}$. Since the molecular region under study extends more than the instantaneous field of view of EVN at $6.7 \mathrm{GHz}\left(\approx 44^{\prime \prime} 6\right.$, using $2 \mathrm{~s}$ of integration time), data were correlated in two passes using two distinct phase centers: $\alpha(\mathrm{J} 2000)=18^{\mathrm{h}} 36^{\mathrm{m}} 12^{\mathrm{s}} .509, \delta(\mathrm{J} 2000)=-7^{\circ} 12^{\prime} 11^{\prime \prime} .14$ and $\alpha(\mathrm{J} 2000)=18^{\mathrm{h}} 36^{\mathrm{m}} 13^{\mathrm{s}} .138, \delta(\mathrm{J} 2000)=-7^{\circ} 12^{\prime} 7^{\prime \prime} \cdot 90$, corresponding to the positions of molecular cores A and C. VLBI (EVN and VLBA) data were calibrated and imaged using the AIPS package, following the standard procedure for VLBI line data (see Moscadelli et al. 2005, for details). 6.7 GHz maser data were self-calibrated using the strongest emission channel (at $113.3 \mathrm{~km} \mathrm{~s}^{-1}$ ).

Centered on each of the two phase centers, we produced naturally weighted maps extended over an area of 3 .' $3 \times 3$ '. 3 and covering the velocity range from 98 to $127 \mathrm{~km} \mathrm{~s}^{-1}$. The CLEAN beam was an elliptical Gaussian with a FWHM size of $14 \times 5$ mas, $\mathrm{PA}=178^{\circ}$. Each channel map was searched for emission above the absolute value of the minimum in the map, which was always greater than $7 \sigma$, with $\sigma$ varying in the range 2-60 mJy beam ${ }^{-1}$. The detected maser spots were fitted with two-dimensional elliptical Gaussians and collected in single "features". A maser feature is considered real if it is detected in at least two contiguous channels (i.e., line-width $>0.6 \mathrm{~km} \mathrm{~s}^{-1}$ ), with a position shift of the intensity peak from channel to channel smaller than the FWHM size. The absolute position of the reference maser spot was determined by mapping the reference channel after calibrating its visibility phase with the corrections derived working with the phase-reference calibrator. The low signal-to-noise of the image and poor cleaning effects limited the accuracy in the knowledge of the absolute position to $\approx 50$ mas in both coordinates.

\subsection{VLBA observations}

\subsection{1. $\mathrm{OH} 1.6 \mathrm{GHz}$ masers}

Using the Very Long Baseline Array (VLBA) ${ }^{3}, \mathrm{G} 24.78+0.08$ was observed at the frequencies of both main-line ${ }^{2} \prod_{3 / 2}, J=$ $3 / 2 \mathrm{OH}$ transitions (1665.4018 and $1667.3590 \mathrm{MHz}$ ), on September 15 and 16, 2003, for $9 \mathrm{~h}$. The phase center was located at $\alpha(\mathrm{J} 2000)=18^{\mathrm{h}} 36^{\mathrm{m}} 12^{\mathrm{s}} .468, \delta(\mathrm{J} 2000)=-7^{\circ} 12^{\prime} 10^{\prime} .63$. The source was observed using a $250 \mathrm{kHz}$ bandwidth divided into 256 spectral channels, providing a total velocity coverage of $45 \mathrm{~km} \mathrm{~s}^{-1}$ and a velocity resolution of $0.176 \mathrm{~km} \mathrm{~s}^{-1}$, in each of the $\mathrm{OH}$ main lines at 1665 and $1667 \mathrm{MHz}$. The observations were performed in phase-reference mode, alternating 4 min scans on the maser source with 1 min scans on the nearby calibrator J1825-0737. Interlapsed every $\approx 80 \mathrm{~min}, 5 \mathrm{~min}$

3 The VLBA is operated by the National Radio Astronomy Observatory (NRAO). 
scans on several continuum sources $(\mathrm{J} 1800+7828, \mathrm{~J} 1642+39$, $\mathrm{J} 2005+7752$ ) were observed for calibration purposes.

The strongest $(\approx 5 \mathrm{Jy})$, spectrally unblended maser spot (at $110.9 \mathrm{~km} \mathrm{~s}^{-1}$ ) of the $1665 \mathrm{MHz}$ maser emission was selected to self-calibrate the data. No signal was recovered in the map constructed with the maser-referenced visibilities of the phase-reference source J1825-0737, and that prevented the determination of the absolute position of the $\mathrm{OH}$ masers. For the $1667 \mathrm{MHz}$ transition, no emission channel was sufficiently strong to allow the determination of the phase corrections, and the data of this transition were discarded.

We produced naturally weighted maps of the $1665 \mathrm{MHz}$ masers extended over an area of $30^{\prime \prime} \times 30^{\prime \prime}$ and covering the velocity range from 103 to $121 \mathrm{~km} \mathrm{~s}^{-1}$. The CLEAN beam was an elliptical Gaussian with a $F W H M$ size of $48 \times 40$ mas, $\mathrm{PA}=22^{\circ}$. The rms noise level on the channel maps was $\sigma \sim$ $30 \mathrm{mJy}$ beam $^{-1}$.

\subsection{2. $\mathrm{H}_{2} \mathrm{O} 22 \mathrm{GHz}$ masers}

Using the VLBA, we observed the $6_{16}-5_{23} \mathrm{H}_{2} \mathrm{O}$ maser line (rest frequency $22235.080 \mathrm{MHz}$ ) at four epochs (4 September and 30 November 2003, 18 February and 19 June 2004), with an observing time of $12 \mathrm{~h}$ per epoch. The observations were performed in phase-reference mode, alternating scans on the maser source and the phase-reference source, J1825-0737, with a switching cycle of $70 \mathrm{~s}$. Interlapsed every $\approx 80 \mathrm{~min}, 3 \mathrm{~min}$ scans on several continuum sources $(\mathrm{J} 1642+39, \mathrm{~J} 1751+09$, $\mathrm{J} 2005+7752$, J2123+05) were observed for calibration purposes. Dual circular polarization was recorded using a $16 \mathrm{MHz}$ bandwidth centered on the LSR velocity of $110 \mathrm{~km} \mathrm{~s}^{-1}$. The data were correlated with the VLBA FX correlator in Socorro (New Mexico) with an integration period of $1 \mathrm{~s}$. The correlator used 1024 spectral channels corresponding to a channel separation of $0.2 \mathrm{~km} \mathrm{~s}^{-1}$. Since previous VLA observations detected water maser emission in correspondence of both $\mathrm{mm}$ cores $\mathrm{A}$ and $\mathrm{C}$, two distinct correlation centers were used: $\alpha(\mathrm{J} 2000)=18^{\mathrm{h}} 36^{\mathrm{m}} 12^{\mathrm{s}} .579, \delta(\mathrm{J} 2000)=-7^{\circ} 12^{\prime} 11^{\prime \prime} \cdot 34$ and $\alpha(\mathrm{J} 2000)=18^{\mathrm{h}} 36^{\mathrm{m}} 13^{\mathrm{s}} .084, \delta(\mathrm{J} 2000)=-7^{\circ} 12^{\prime} 07^{\prime \prime} \cdot 32$, pointing towards the VLA $22 \mathrm{GHz}$ emission barycenter either in core A or C. To self-calibrate the line data correlated using the first and the second phase center, we used two different, strong, spectrally isolated maser spots, one emitting at $112.3 \mathrm{~km} \mathrm{~s}^{-1}$, and the other one at $114.5 \mathrm{~km} \mathrm{~s}^{-1}$, respectively.

Inside the VLBA field of view centered on either of the two correlator centres, six distinct VLA water maser emission centres were detected, i.e. "A1", "A2", "A/B", "B" and "E" towards core A, and " $\mathrm{C}$ " towards core $\mathrm{C}$ (see Fig. 4, upper right panel). In correspondence of each of the six VLA emission centers, we produced VLBA maps covering a sky area and a LSR velocity range suitable to recover all the detected VLA maser features. The CLEAN beam was an elliptical Gaussian with a $F W H M$ size (slightly varying from epoch to epoch) of $\approx 1.5 \times$ 0.4 mas, $\mathrm{PA}=170^{\circ}$. At each observing epoch, the rms noise level on the channel maps, $\sigma$, varied over a similar range of values, $4-40 \mathrm{mJy}^{\mathrm{beam}}{ }^{-1}$. For a detailed description of the procedures for Gaussian fitting and for collecting maser spots into "features", of criteria for establishing correspondence over time for persistent features, and of the derivation of feature proper motions, we refer to Moscadelli et al. (2006) and Goddi \& Moscadelli (2006). The absolute position of the reference maser spot was determined by mapping the reference channel after calibrating its visibility phase with the corrections derived working with the phase-reference source. The uncertainty in the absolute position, calculated taking the ratio between the undeconvolved FWHM size of the main peak and the signal-to-noise ratio of the map, was 0.15 mas in both coordinates. The error in the absolute position of a given maser feature (reported in Tables 5 and 6 ) was calculated by taking the root sum squared of the uncertainties in both the relative position of the feature and the absolute position of the reference spot. The accuracy in the absolute positions was good enough to derive (via a linear fit of the variation of positions with time) accurate $(S / N \geq 3)$ proper motions for many maser features, but unsufficient to estabilish whether the trend of positions with time were represented better by a quadratic rather than a linear fit. Therefore we could not ascertain the presence of feature accelerations.

\section{Results}

\subsection{Class I $\mathrm{CH}_{3} \mathrm{OH}$ masers}

We have identified 37 and 7 distinct maser features at $44 \mathrm{GHz}$ and $95 \mathrm{GHz}$, respectively. The maser feature properties $\left(V_{\mathrm{LSR}}\right.$, flux density, RA and Dec peak position) are presented in Table 1.

Figure 1 presents the maps of the $\mathrm{CS}(3-2)$ line (Cesaroni et al. 2003) and the ${ }^{12} \mathrm{CO}(1-0)$ wing emission (Furuya et al. 2002) overlaid on the positions of the 95 and $44 \mathrm{GHz}$ methanol masers. $44 \mathrm{GHz}$ methanol masers are found to be distributed within an area of about $20^{\prime \prime} \times 20^{\prime \prime}$, and, although several spots are isolated and scattered across this area, most of $44 \mathrm{GHz}$ features concentrate in three main clusters. The three $44 \mathrm{GHz}$ clusters correspond in position with the two main peaks of the CS(3-2) emission, i.e. with molecular cores A and C, and with a weaker CS(3-2) spur to the northwest of core C. The spatial distribution of $95 \mathrm{GHz}$ masers is less scattered than that of $44 \mathrm{GHz}$ masers and more closely associated with the CS(3-2) emission features. Each one of the detected $95 \mathrm{GHz}$ maser spots is aligned in position with a strong $44 \mathrm{GHz}$ spot in one of the three maser clusters. Towards core A and the CS(3-2) spur, the Class I methanol maser features present an elongated distribution and a spread of LSR velocities of $7-10 \mathrm{~km} \mathrm{~s}^{-1}$, whereas the methanol maser features clustered towards core $\mathrm{C}$ show an approximately ellipsoidal distribution with a lower spread in LSR velocities, $\approx 5 \mathrm{~km} \mathrm{~s}^{-1}$. It is interesting to note that the direction of elongation of the $44 \mathrm{GHz} \mathrm{CH} \mathrm{OH}_{3} \mathrm{Omasers}$ in the core A cluster is approximately parallel to the axis of the ${ }^{12} \mathrm{CO}(1-0)$ molecular outflow.

\subsection{Class $/ / \mathrm{CH}_{3} \mathrm{OH}$ masers}

Fourteen distinct $\mathrm{CH}_{3} \mathrm{OH} 6.7 \mathrm{GHz}$ maser features have been detected towards core A. Table 2 summarizes the derived observational parameters. Figure 2 reports the map of the $\mathrm{CH}_{3} \mathrm{CN}$ (12-11) line LSR velocity overlaid on the contour map of the $1.4 \mathrm{~mm}$ continuum emission towards G24 A1 and G24 A2 (Beltrán et al. 2004). The absolute positions (accurate within 50 mas) of the $6.7 \mathrm{GHz}$ methanol masers are also reported as filled black circles (Fig. 2, panel a). The $6.7 \mathrm{GHz}$ maser emission stems from two distinct groups of features, each one associated to either of the mm subcores A1 and A2. Both groups of maser features are elongated along a direction that agrees well with the axis of the LSR velocity gradient measured in the $\mathrm{CH}_{3} \mathrm{CN}$ $1.4 \mathrm{~mm}$ line.

Panels b and c of Fig. 2 show the LSR velocities of the 6.7 GHz maser features, associated respectively with the A2 and A1 subcores. Across each maser group, the variation of LSR velocities agrees with the velocity gradient observed using 


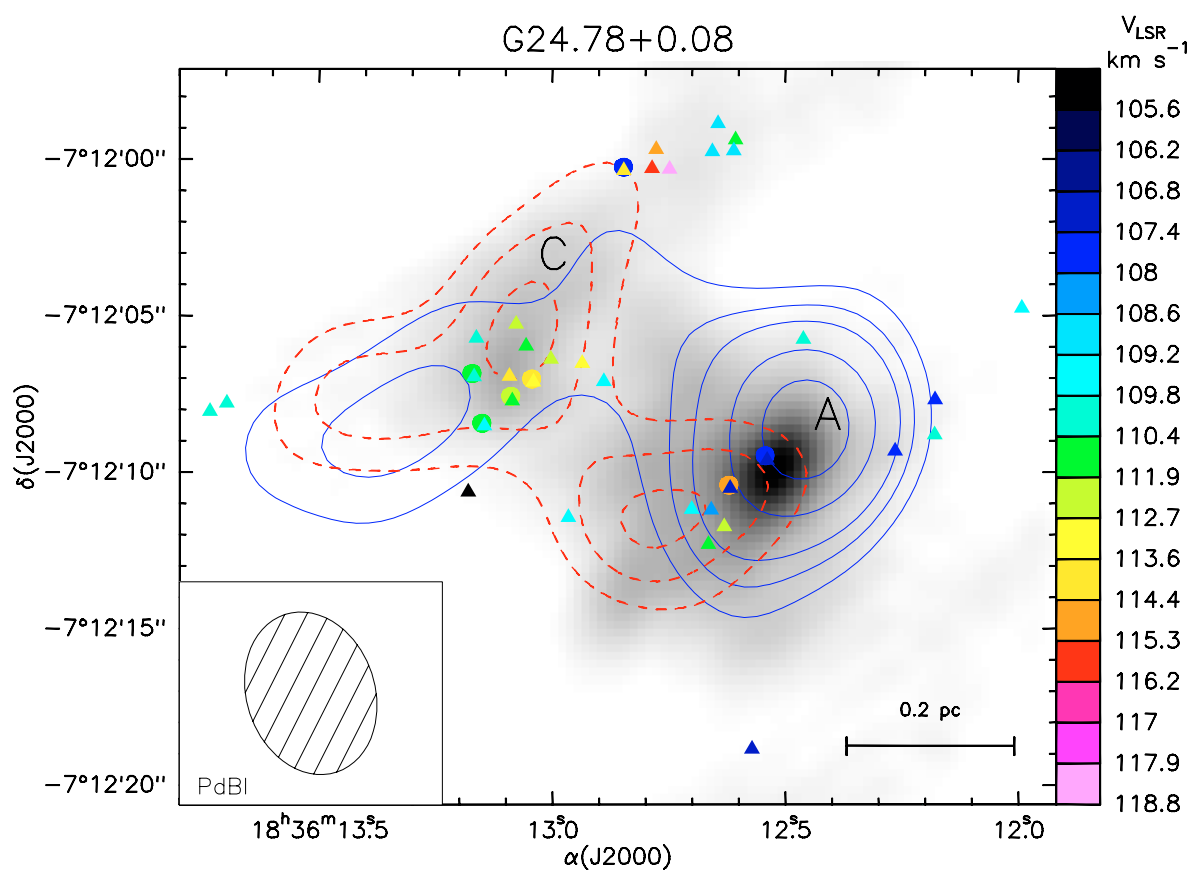

Fig. 1. Overlay of the BIMA $95 \mathrm{GHz}$ (filled circles) and the VLA $44 \mathrm{GHz}$ (filled triangles) Class I $\mathrm{CH}_{3} \mathrm{OH}$ masers with the $\mathrm{CS}(3-2)$ integrated map (grey scale) obtained with the Nobeyama Millimeter Array by Cesaroni et al. (2003) and with blue (solid lines) and red (dashed lines) lobes of ${ }^{12} \mathrm{CO}(1-0)$ wing emission observed with PdBI by Furuya et al. (2002); different colors are used to indicate the maser LSR velocities, according to the colored scale on the right-hand side of the plot.
Table 2. Parameters of Class II $\mathrm{CH}_{3} \mathrm{OH}$ maser features detected with the EVN at $6.7 \mathrm{GHz}$.

\begin{tabular}{ccccc}
\hline \hline Core & $\begin{array}{c}V_{\text {LSR }} \\
\left(\mathrm{km} \mathrm{s}^{-1}\right)\end{array}$ & $\begin{array}{c}F_{\text {int }} \\
(\mathrm{Jy})\end{array}$ & $\begin{array}{c}\Delta \alpha \\
(\mathrm{mas})\end{array}$ & $\begin{array}{c}\Delta \delta \\
(\mathrm{mas})\end{array}$ \\
\hline $\mathrm{A} 1$ & 113.3 & 34.8 & 0 & 0 \\
$\mathrm{~A} 1$ & 114.1 & 7.3 & $-75.7 \pm 0.2$ & $98.4 \pm 0.6$ \\
$\mathrm{~A} 1$ & 114.7 & 2.9 & $-140.3 \pm 0.2$ & $77.6 \pm 0.4$ \\
$\mathrm{~A} 1$ & 116.6 & 0.2 & $-254.8 \pm 0.2$ & $-116.8 \pm 0.5$ \\
$\mathrm{~A} 1$ & 107.5 & 3.8 & $-436.7 \pm 0.5$ & $-402.0 \pm 0.4$ \\
$\mathrm{~A} 1$ & 107.8 & 1.6 & $-408.2 \pm 0.3$ & $-408.6 \pm 0.6$ \\
$\mathrm{~A} 1$ & 108.3 & 1.3 & $-426.9 \pm 0.2$ & $-404.1 \pm 0.4$ \\
$\mathrm{~A} 1$ & 107.5 & 9.1 & $-442 \pm 1.0$ & $-404.9 \pm 0.7$ \\
$\mathrm{~A} 2$ & 112.0 & 2.5 & $-1155.0 \pm 0.2$ & $995.8 \pm 0.6$ \\
$\mathrm{~A} 2$ & 112.1 & 5.9 & $-1389 \pm 2$ & $1034.0 \pm 0.5$ \\
$\mathrm{~A} 2$ & 109.5 & 1.9 & $-1315.0 \pm 0.2$ & $701.6 \pm 0.4$ \\
$\mathrm{~A} 2$ & 110.4 & 7.8 & $-1323.0 \pm 0.2$ & $695.0 \pm 0.5$ \\
$\mathrm{~A} 2$ & 108.5 & 0.6 & $-1542.0 \pm 0.2$ & $743.9 \pm 0.5$ \\
$\mathrm{~A} 2$ & 106.4 & 0.3 & $-1448.0 \pm 0.2$ & $552.6 \pm 0.5$ \\
\hline
\end{tabular}

Note. For each identified feature, Col. 1 gives the harboring subcore; Cols. 2 and 3 the LSR velocity and the integrated flux density of the highest-intensity channel; Cols. 4 and 5 the relative positional (RA and Dec) offsets (with the associated errors) evaluated with respect to the reference feature. The derived absolute position of the reference feature is $\alpha(J 2000)=18^{\mathrm{h}} 36^{\mathrm{m}} 12 \mathrm{~s} .563, \delta(J 2000)=-07^{\circ} 12^{\prime} 10^{\prime} .792$, with an uncertainty of $\approx 50$ mas in both coordinates.

the $\mathrm{CH}_{3} \mathrm{CN}$ (12-11) line in the harboring subcore, with the redshifted velocities to the NE and the blueshifted ones to the SW. The methanol maser features are distributed over regions $\sim 3$ times smaller $(0.023$ and $0.02 \mathrm{pc})$ and cover a LSR velocity range $\sim 3$ times larger $\left(9.1\right.$ and $\left.5.7 \mathrm{~km} \mathrm{~s}^{-1}\right)$ than the emission of the $\mathrm{CH}_{3} \mathrm{CN}$ thermal line in both subcores. We will comment on this result in Sect. 5.1.

\section{3. $\mathrm{OH}$ masers}

Only 6 distinct $\mathrm{OH} 1665 \mathrm{MHz}$ maser features were detected, whose observed properties ( $V_{\mathrm{LSR}}$, flux density, RA and Dec peak position) are presented in Table 3 . Figure 3 shows that the $\mathrm{OH}$ maser features are concentrated in two clusters, the first one (comprising features labelled 1, 2, 3, and 4) located towards the NW corner of the plotted area, and the second one (comprising features labelled 5 and 6) detected towards the SE corner of the plotted area. The sky-projected separation between the two clusters is $\sim 1^{\prime \prime}$, corresponding to $\sim 7700$ AU at a distance of $7.7 \mathrm{kpc}$. Except for a single feature (with label number 5), all $\mathrm{OH}$ masers have LSR velocities blueshifted by a few kilometers per second.

As noted before, at $1.6 \mathrm{GHz}$ we could not estimate the absolute position of the maser emission. However, the $1665 \mathrm{MHz}$ maser spectrum derived with our VLBA observations is similar to the VLA spectrum of Forster \& Caswell (1989). If most of the $\mathrm{OH}$ maser features were persistent between the VLA and VLBA observations, then the $1665 \mathrm{MHz}$ VLBA emission would originate from the same location of the VLA detections, i.e. from the molecular core A.

\section{4. $\mathrm{H}_{2} \mathrm{O}$ masers}

19 distinct $22.2 \mathrm{GHz}$ water maser features have been detected with the VLA in the G24.78+0.08 SFR (the observational parameters are presented in Table 4). The LSR velocities of the $22.2 \mathrm{GHz}$ water masers vary over a larger velocity range (from 92 to $127 \mathrm{~km} \mathrm{~s}^{-1}$ ) as compared with other maser species. VLA water masers are found to be distributed within an area of about $10^{\prime \prime} \times 10^{\prime \prime}$, and clustered into 8 regions. In Fig. 4 (upper left panel) these are indicated with "A1", "A2", "A/B", "B", "C", "E", "F", and "G". Clusters "A1", "A2", "B", and "C" are associated with the homonymous ( $\mathrm{mm}$ ) cores, "A/B" is found between cores A and B, whereas clusters "E", "F", and "G" apparently are not associated with any known mm continuum source in the G24.78+0.08 SFR. Cluster "C" presents an arc-shaped structure, with a curvature radius of $\sim 200$ mas (corresponding to $\sim 1500$ AU at $7.7 \mathrm{kpc}$ ).

By observing G24.78+0.08 at $22.2 \mathrm{GHz}$ with the VLBA in phase-reference mode at four different epochs, absolute positions and proper motions of the water maser features have been derived. In the whole mapped region, 246 distinct water maser features have been detected in at least one of the four epochs, 


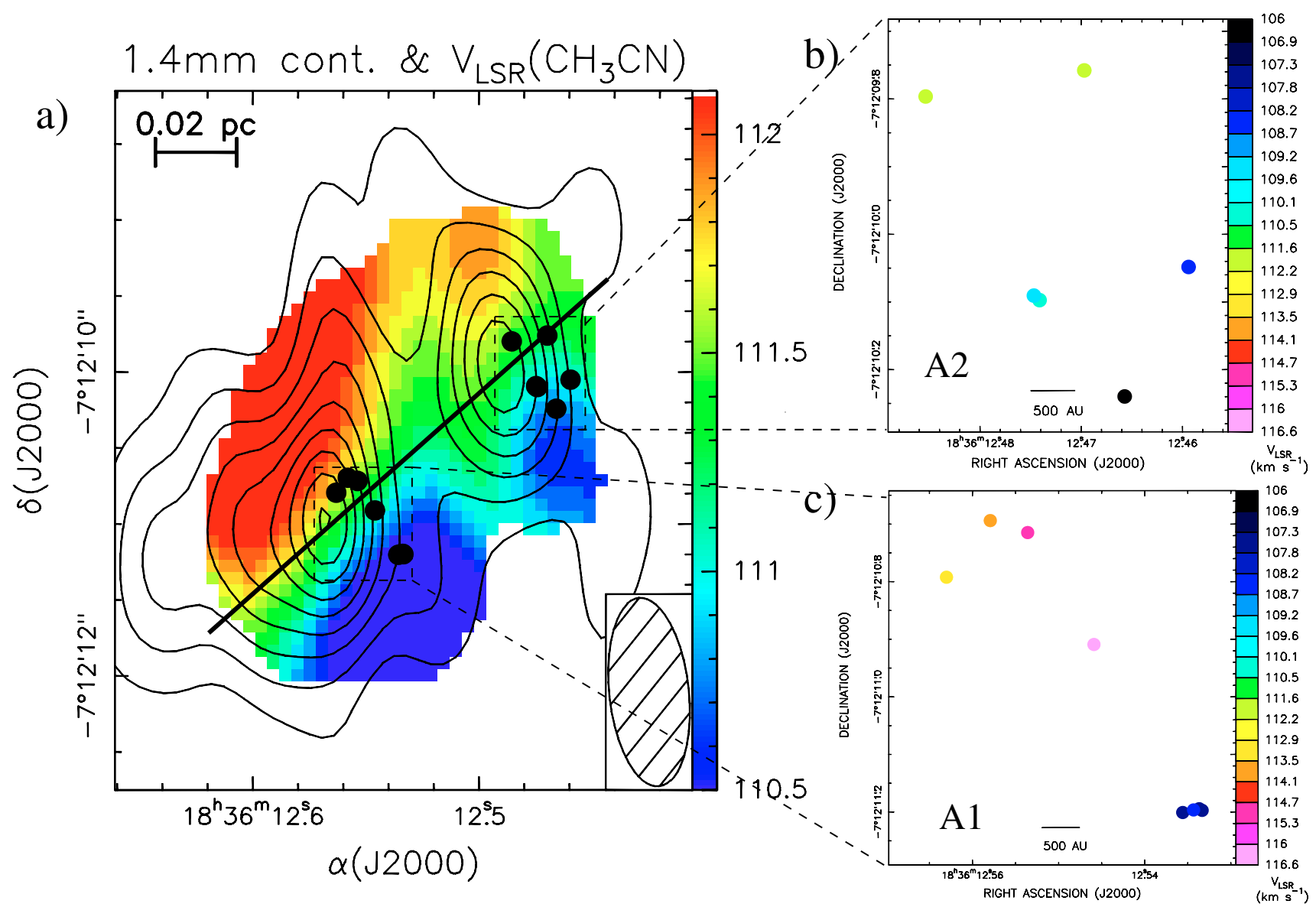

Fig. 2. a) The positions of the $\mathrm{CH}_{3} \mathrm{OH} 6.7 \mathrm{GHz}$ masers (filled black circles) are shown on top of the map of the $\mathrm{CH}_{3} \mathrm{CN}(12 \rightarrow 11)$ line peak velocity towards G24 A, with the contour levels in kilometers per seconds indicated in the wedge to the right of the panel (Beltrán et al. 2004); overlaid on the $\mathrm{CH}_{3} \mathrm{CN}$ velocity map, it is shown the contour map of the $1.4 \mathrm{~mm}$ continuum emission (solid lines); the straight black line represents the outflow axis. The insert in the lower right corner of the panel shows the PdBI beam at $1.4 \mathrm{~mm}$. b) and c) Positions and line of sight velocities of the $6.7 \mathrm{GHz}$ maser features observed towards the subcore A2 and A1, respectively, with the colors denoting different LSR velocities, according to the scale on the right-hand side of the panels.

Table 3. Parameters of the $\mathrm{OH} 1665 \mathrm{MHz}$ maser features detected with the VLBA.

\begin{tabular}{ccccc}
\hline \hline Feature & $\begin{array}{c}V_{\text {LSR }} \\
\left(\mathrm{km} \mathrm{s}^{-1}\right)\end{array}$ & $\begin{array}{c}F_{\text {int }} \\
(\mathrm{Jy})\end{array}$ & $\begin{array}{c}\Delta \alpha \\
(\mathrm{mas})\end{array}$ & $\begin{array}{c}\Delta \delta \\
(\mathrm{mas})\end{array}$ \\
\hline 1 & 110.9 & 5.5 & 0 & 0 \\
2 & 105.7 & 0.5 & $-201 \pm 1$ & $-361 \pm 3$ \\
3 & 107.3 & 0.8 & $-6 \pm 1$ & $-506 \pm 3$ \\
4 & 109.1 & 0.5 & $-94 \pm 9$ & $-518 \pm 8$ \\
5 & 112.2 & 1.9 & $1239 \pm 1$ & $-985 \pm 4$ \\
6 & 105.9 & 1.1 & $759 \pm 1$ & $-1439 \pm 2$ \\
\hline
\end{tabular}

Note. For each identified feature, Col. 1 gives the label number; Cols. 2 and 3 the LSR velocity and the integrated flux density of the highestintensity channel; Cols. 4 and 5 the relative positional (RA and Dec) offsets (with the associated errors) evaluated with respect to the reference feature.

and 70 of these were persistent over three or four epochs, allowing the measurement of their proper motions. For each of the six VLA centers ("A1", "A2", "B", "A/B", "C", and "E"; Fig. 4, upper left panel) mapped with the VLBA, Tables 5 and 6 give the parameters of the identified features. Owing to the much higher angular $\left(\sim 00^{\prime} 0008\right.$ vs. $\left.\sim 00^{\prime} 2\right)$ and velocity $\left(0.2\right.$ vs. $\left.0.66 \mathrm{~km} \mathrm{~s}^{-1}\right)$ resolution of the VLBA data, most of the VLA single maser features unfold into clusters of features at the VLBA scales,

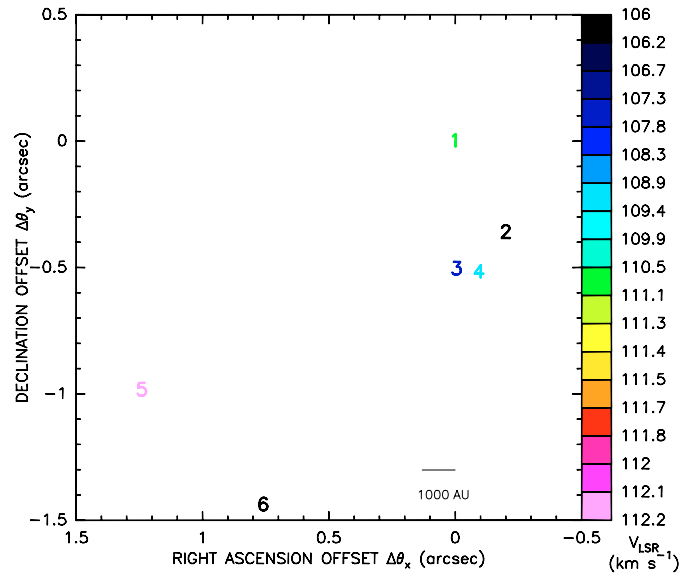

Fig. 3. Relative positions (label numbers) and LSR velocities (colours) of $\mathrm{OH} 1665 \mathrm{MHz}$ maser features detected with the VLBA.

showing arcuate or linear structures. Compared to the VLA, the larger frequency coverage of the VLBA observations allowed to detect maser emission over a much wider interval of LSR velocities, ranging from 34.5 to $127.3 \mathrm{~km} \mathrm{~s}^{-1}$.

In the following, the spatial distribution and the kinematics of the VLBA maser clusters are described individually. 
Table 4. Parameters of $\mathrm{H}_{2} \mathrm{O}$ maser features detected with the VLA.

\begin{tabular}{ccccc}
\hline \hline Cluster & $\begin{array}{c}V_{\mathrm{LSR}} \\
\left(\mathrm{km} \mathrm{s}^{-1}\right)\end{array}$ & $\begin{array}{c}F_{\text {int }} \\
(\mathrm{Jy})\end{array}$ & $\begin{array}{c}\alpha(\mathrm{J} 2000) \\
(\mathrm{h} \mathrm{m} \mathrm{s})\end{array}$ & $\begin{array}{c}\delta(\mathrm{J} 2000) \\
\left({ }^{\circ}{ }^{\prime \prime}\right)\end{array}$ \\
\hline & & & & \\
$\mathrm{A} 1$ & 112.3 & 305.7 & 183612.549 & -071210.78 \\
$\mathrm{~A} 1$ & 97.7 & 0.06 & 183612.545 & -071210.80 \\
$\mathrm{~A} 1$ & 127.1 & 0.06 & 183612.546 & -071210.79 \\
$\mathrm{~A} 2$ & 105.6 & 10.3 & 183612.443 & -071210.15 \\
$\mathrm{~A} / \mathrm{B}$ & 93.1 & 0.6 & 183612.611 & -071211.99 \\
$\mathrm{~B}$ & 103.8 & 0.4 & 183612.646 & -071213.68 \\
$\mathrm{~B}$ & 92.6 & 0.2 & 183612.658 & -071213.54 \\
$\mathrm{C}$ & 114.6 & 5.8 & 183613.293 & -071207.29 \\
$\mathrm{C}$ & 122.5 & 2.1 & 183612.285 & -071207.17 \\
$\mathrm{C}$ & 114.6 & 5.0 & 183613.280 & -071207.54 \\
$\mathrm{C}$ & 108.0 & 3.5 & 183613.283 & -071207.50 \\
$\mathrm{C}$ & 106.0 & 1.1 & 183613.277 & -071207.16 \\
$\mathrm{C}$ & 95.7 & 0.8 & 183613.260 & -071207.12 \\
$\mathrm{C}$ & 97.9 & 0.8 & 183613.278 & -071207.16 \\
$\mathrm{C}$ & 116.7 & 0.6 & 183613.266 & -071207.17 \\
$\mathrm{E}$ & 102.8 & 0.9 & 183612.336 & -071211.48 \\
$\mathrm{E}$ & 94.2 & 0.8 & 183613.337 & -071211.48 \\
$\mathrm{~F}$ & 109.8 & 0.5 & 183612.419 & -071208.03 \\
$\mathrm{G}$ & 112.1 & 3.5 & 183612.408 & -071215.52 \\
& & & &
\end{tabular}

Note. For each identified feature, Col. 1 indicates the harboring (sub)cluster; Cols. 2 and 3 the LSR velocity and the integrated flux density of the highest-intensity channel; Cols. 4 and 5 the positional (RA and $\mathrm{Dec}$ ) coordinates.

\subsubsection{Cluster "A1"}

The subcore A1 contains the strongest water maser features of the entire $\mathrm{G} 24.78+0.08$ region, with intensities ranging from $\approx 0.05$ to $\approx 100 \mathrm{Jy} \mathrm{beam}^{-1}$. We detected a total of 96 maser features, and for 34 of these, persistent over three or four epochs, proper motions were measured. Maser emission arises from two distinct regions named "A1N" and "A1S" (Fig. 4, bottom right panel), found in the $\mathrm{NE}$ and $\mathrm{SW}$ corner of the plotted area, respectively. The "A1N" subcluster comprises the majority of the features (88) detected in the "A1" cluster, and its most intense features distribute along an EW-oriented line (with length $\approx 100$ mas) and along an arc-shaped structure (of diameter $\approx 50$ mas). With a few exceptions, most of the maser features present a shift in LSR velocity, $\left|V_{\mathrm{LSR}}-V_{\text {sys }}\right|$, from the systemic velocity of the region $\left(V_{\text {sys }}=111 \mathrm{~km} \mathrm{~s}^{-1}\right)$, less than $5 \mathrm{~km} \mathrm{~s}^{-1}$. The measured absolute proper motions, with amplitudes in the range $20-60 \mathrm{~km} \mathrm{~s}^{-1}$, have directions roughly perpendicular to the linear and arc-like spatial distributions of the maser features.

Subcluster "A1S" consists of a compact (size $\approx 30$ mas) group of eight maser features located $\approx 600$ mas towards SW from the "A1N" subcluster. The LSR velocities of the "A1S" features are slightly blueshifted (in the range $108-110 \mathrm{~km} \mathrm{~s}^{-1}$ ) with respect to $V_{\text {sys }}$. No proper motion has been measured for features of this subcluster.

\subsubsection{Cluster "A2"}

In subcore A2, rather weak water maser emission has been detected with the VLBA. Panel A2 of Fig. 4 shows the spatial and velocity distribution of the few (7) detected maser features. The water features present a linear distribution of size $\approx 0.3$ oriented along the EW direction. The LSR velocities, ranging from 105 to $107 \mathrm{~km} \mathrm{~s}^{-1}$, are blueshifted with respect to $V_{\text {sys }}$. Although the measured proper motions have large relative errors, it can be noted that the overall direction of motion of the maser features is towards N-NW.

\subsubsection{Cluster "B"}

A small group of six water maser features has been detected $1^{\prime \prime}-2^{\prime \prime}$ north from the peak of the ionized continuum emission in core B. These features show an elongated spatial distribution of size $\approx 0$.' 6 , whose major axis is oriented close to the EW direction (Fig. 4, panel B). The LSR velocities of these features are significantly blueshifted with respect to $V_{\text {sys }}$, ranging from 92 to $106 \mathrm{~km} \mathrm{~s}^{-1}$. The two measured proper motions have large $\left(\geq 100 \mathrm{~km} \mathrm{~s}^{-1}\right)$ amplitudes and are directed towards N-NW.

\subsubsection{Cluster "C"}

The VLBA maser cluster "C" is located close to the center of the $\mathrm{mm}$ core $\mathrm{C}$. We identified 117 maser features and measured proper motions for 23 of them, whose emission was persistent over three or four epochs. While towards core A water maser emission is detected across a LSR velocity range within $\pm 20 \mathrm{~km} \mathrm{~s}^{-1}$ from $V_{\text {sys }}\left(\left|V_{\mathrm{LSR}}-V_{\text {sys }}\right| \leq 20 \mathrm{~km} \mathrm{~s}^{-1}\right)$, towards core $\mathrm{C}$ water features emit across a much larger range of blueshifted velocities, extending up to $\approx 80 \mathrm{~km} \mathrm{~s}^{-1}$ below $V_{\text {sys }}$. Correspondingly, the measured absolute proper motions in core $\mathrm{C}$, with amplitudes varying in the range $80-200 \mathrm{~km} \mathrm{~s}^{-1}$, are significantly higher than those observed for maser features of core A. Based on the spatial and the 3D velocity distribution of the maser features, cluster " $C$ " can be divided into three subclusters, named "C1", "C2", and "C3" (see Fig. 4, panel C). Subcluster "C1" consists of a NE-SW elongated distribution of maser features seen towards the SE corner of the plotted area. The direction of the measured proper motions is almost parallel to the elongation of the masers' distribution. The features of subcluster "C1" present moderately redshifted velocities, mostly within $10 \mathrm{~km} \mathrm{~s}^{-1}$ from $V_{\text {sys }}$.

In the subcluster " $\mathrm{C} 3$ ", located roughly at the center of the plotted area, more than 60 features are grouped within a small area of diameter $\approx 1000$ AU. For eleven features of this subcluster the proper motions have been accurately measured, which all are nearly parallel and oriented along a direction at close angle with the direction of motion of features in subcluster " $\mathrm{C} 1$ ". The majority of the $\mathrm{H} 2 \mathrm{O}$ maser features constituting the "C3" subcluster are blueshifted more than $30 \mathrm{~km} \mathrm{~s}^{-1}$ with respect to $V_{\text {sys }}$.

The subcluster "C2" is a compact (diameter $\leq 1000 \mathrm{AU}$ ) group of features situated roughly midway between the subcluster "C3" and the NE end of the "C1" subcluster. The "C2" subcluster presents a SE-NW elongated spatial distribution with features to the SE (NW) being redshifted (blueshifted) by up to $\approx 20 \mathrm{~km} \mathrm{~s}^{-1}$ with respect to $V_{\text {sys. }}$. The measured absolute proper motions indicate that features located at opposite ends of the "C2" linear distribution have divergent motions, with skyprojected directions varying by $\approx 90^{\circ}$ across the subcluster.

\subsubsection{Clusters "A/B" and " $E$ "}

The VLBA water maser clusters "A/B" and "E" have a skyprojected distance $\geq 2$ " -3 " from the position of the nearest detected YSOs.

Maser features of cluster "A/B" are found midway between cores A1 and B. They are grouped into two nearby subclusters, having a sky-projected distance of only $\approx 16$ mas (Fig. 4, panel A/B). The LSR velocities of maser features are 

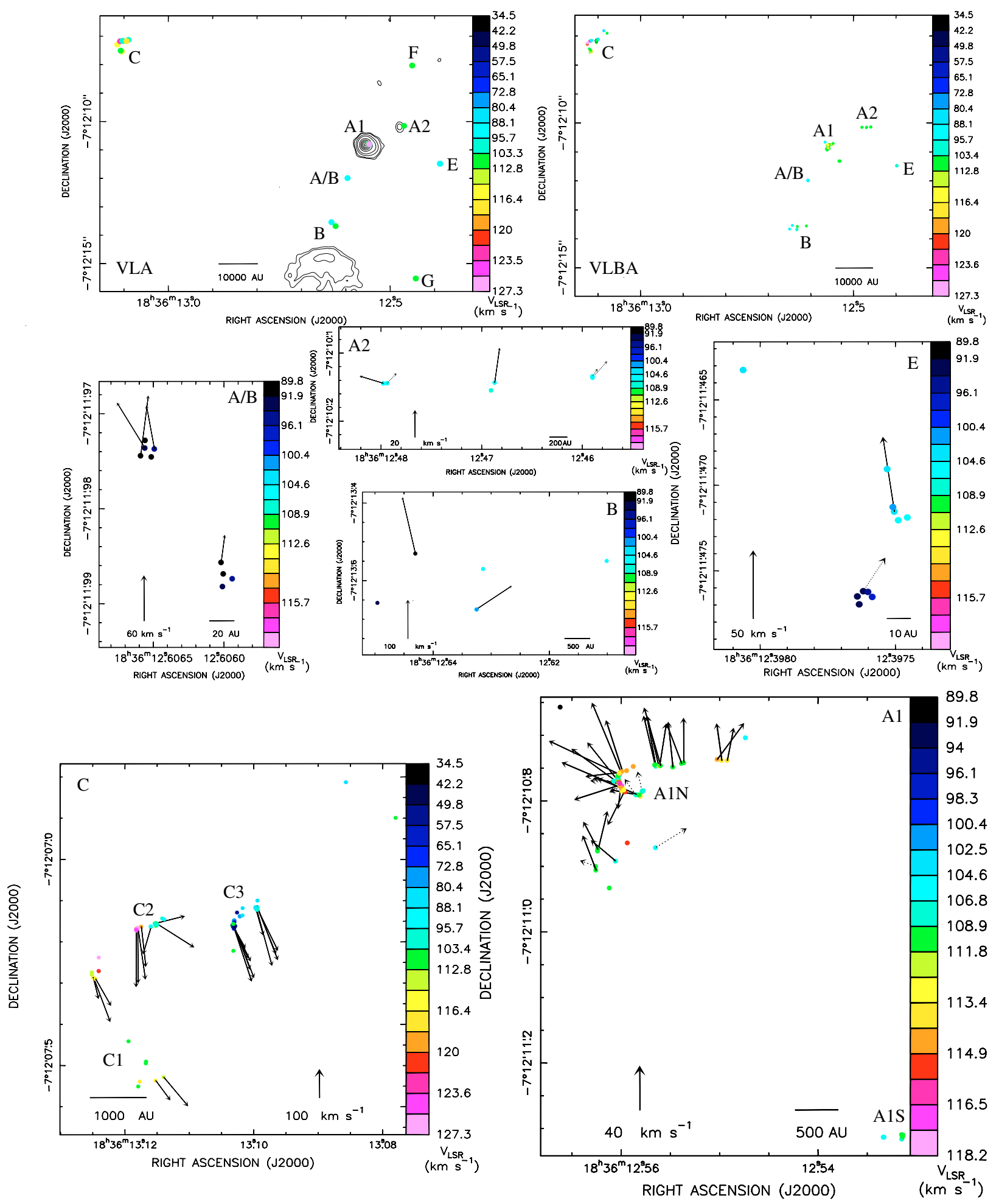

Fig. 4. Position and velocities of the water maser features detected with VLA and VLBA. Upper panels: the left and right upper panels show the spatial distribution of the maser features detected using respectively the VLA and the VLBA arrays. The contour map in the left upper panel shows the three continuum sources (A1, A2 and B) detected towards the G24 core with the VLA-B array in the $1.3 \mathrm{~cm}$ band. The dot colors denote different LSR velocities, according to the scale on the right-hand side of the panels. The VLA and/or VLBA maser emission originates from distinct centers, labelled with the letters "A1", "A2", "A/B", "B", "C", "E", "F", and "G". VLA regions "F" and "G", falling too distant $\left(\geq 3^{\prime \prime}\right)$ from the VLBA correlator position towards the $\mathrm{mm}$ core A, are not mapped with the VLBA. Middle and lower panels: middle and lower panels show the detailed spatial and velocity distribution of the maser features detected in each of the six VLBA maser clusters. Each panel is named after the maser cluster to which it refers. Different colors indicate the variation of the maser LSR velocities, according to the colour scale on the right-hand side of the panel. The measured feature absolute proper motions are indicated with arrows, using dotted arrows to denote the most uncertain measurements; the proper motion amplitude scale is given at the bottom of each panel. Lower panels show that the VLBA maser clusters "A1" and "C" can be further sorted into subclusters "A1N" and "A1S", and subclusters "C1", "C2" and "C3", respectively. 


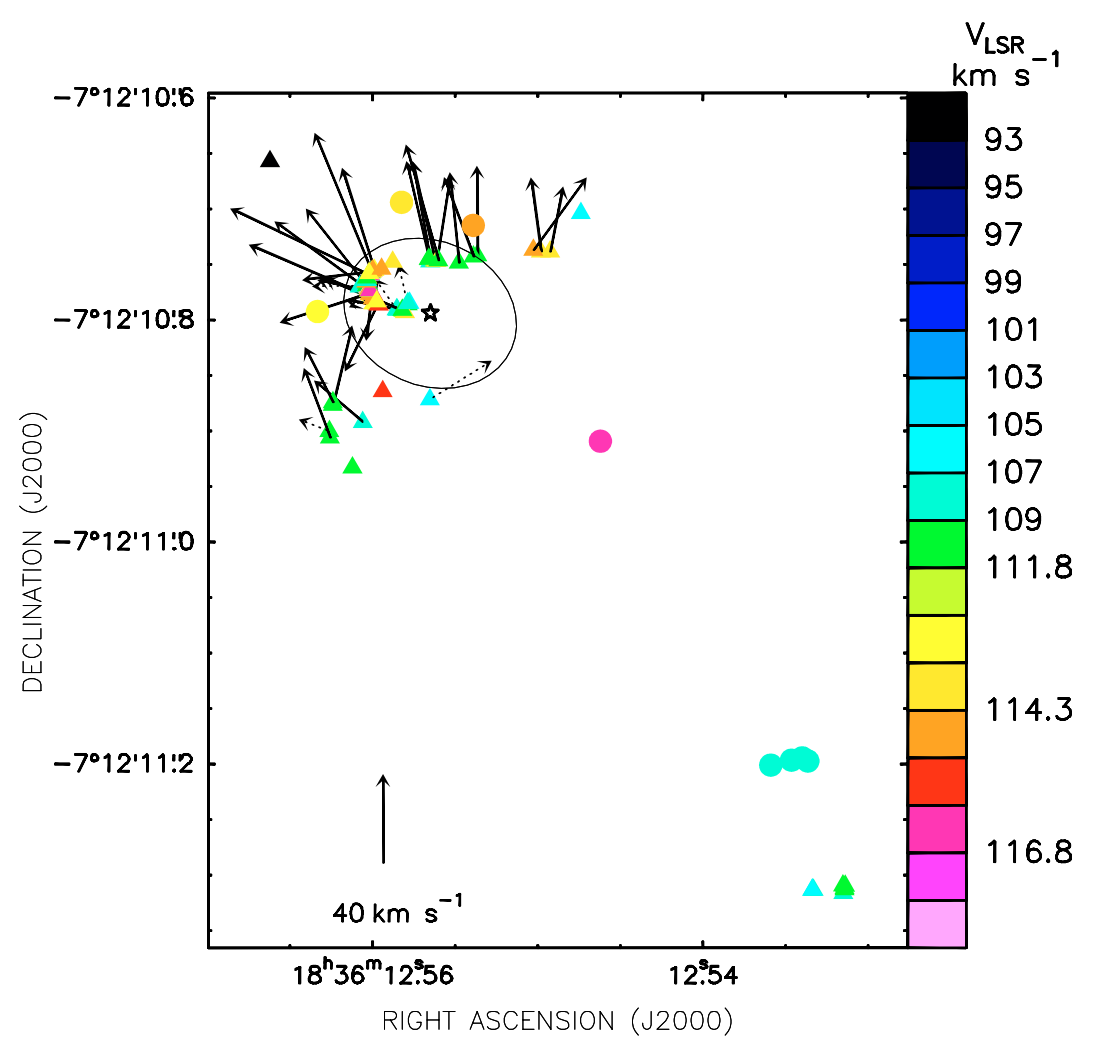

Fig. 5. Comparison of the distribution of the VLBA $22.2 \mathrm{GHz}$ water (triangles) and EVN $6.7 \mathrm{GHz}$ methanol (dots) maser features in the mm subcore G24 A1. The colors denote different LSR velocities, according to the scale on the right-hand side of the panel. Black arrows show the measured absolute proper motions of water maser features, using dotted arrows to denote the most uncertain measurements; the amplitude scale for proper motions is reported at the bottom of the panel. The star symbol and the ellipse show respectively the position and the deconvolved FWHM size of the G24 A1 continuum source observed recently in the $1.3 \mathrm{~cm}$ band with the VLA by Beltrán et al. (2007).

significantly blueshifted and vary in the range $90-95 \mathrm{~km} \mathrm{~s}^{-1}$. The measured proper motions indicate that both subclusters are moving northward with high velocities, in the range $40-80 \mathrm{~km} \mathrm{~s}^{-1}$.

Cluster "E" consists of a group of 11 maser features, found to the west of the mm-core A. The maser emission originates from three small subclusters distributed approximately along the $\mathrm{N}-\mathrm{S}$ direction across a region of $\approx 15$ mas size (Fig. 4, panel E). The LSR velocities of the maser features are blueshifted and vary in the range $94-105 \mathrm{~km} \mathrm{~s}^{-1}$. We measured proper motions of two features belonging to different subclusters, and both are directed towards N-NW.

\section{22.2 GHz water and 6.7 GHz methanol maser association}

Figures 5 and 6 show that the $6.7 \mathrm{GHz}$ methanol and $22.2 \mathrm{GHz}$ water masers have similar positions and LSR velocities towards both subcore G24 A1 and G24 A2. In both subcores the diameter of the maser emitting region is only $\approx 5000-6000 \mathrm{AU}$, and it can be reasonably assumed that a single event of $\mathrm{YSO}(\mathrm{s})$ formation is responsible for exciting and determining the kinematics of both maser species. This result indicates that $22.2 \mathrm{GHz}$ water and $6.7 \mathrm{GHz}$ methanol masers can coexist at a given evolutionary stage of massive star-formation. A similar evidence has been recently found also in the massive star-forming region AFGL 5142 (Goddi et al. 2007), in which $22.2 \mathrm{GHz}$ water and $6.7 \mathrm{GHz}$ methanol masers are observed to originate from the same area (of diameter $\leq 1000 \mathrm{AU}$ ) around an UCHII region. To our knowledge, AFGL 5142 and G24 A1 and G24 A2, reported in this paper, are so far the only sources for which $6.7 \mathrm{GHz}$ methanol and
22.2 GHz water maser association on scales of 10-100 AU has been certified by means of phase-referenced VLBI observations.

Even if the two maser species are associated with the same YSO(s), it is likely that they trace different environments. This is suggested by the maser excitation models predicting different physical conditions for the methanol and water maser transitions. $\mathrm{H}_{2} \mathrm{O}$ maser models explain the excitation by collisional pumping with $\mathrm{H}_{2}$ molecules within hot $(\gtrsim 400 \mathrm{~K})$ shocked layers of gas behind both high-velocity $\left(\geq 40-50 \mathrm{~km} \mathrm{~s}^{-1}\right)$ dissociative (J-type) (Elitzur et al. 1989) and slow $\left(\leq 40-50 \mathrm{~km} \mathrm{~s}^{-1}\right)$ nondissociative (C-type) (Kaufman \& Neufeld 1996) shocks, propagating in dense regions $\left(\mathrm{H}_{2}\right.$ pre-shock density $\left.\geq 10^{6}-10^{7} \mathrm{~cm}^{-3}\right)$. Instead current $\mathrm{CH}_{3} \mathrm{OH}$ excitation models (Cragg et al. 2005) predict that methanol maser emission is produced by radiative pumping in moderately warm regions $(\sim 100-200 \mathrm{~K})$ with $\mathrm{H}_{2}$ densities in the range $10^{6}-10^{9} \mathrm{~cm}^{-3}$. Therefore, even though comparably high densities are required both for water and Class II methanol maser action, strong 6.7 GHz methanol masers can be produced at gas temperature significantly lower than that of the shocked layers of gas harboring water maser emission.

To test the hypothesis that the water and methanol masers trace different regions around the YSO responsible for their excitation, one can compare the average distance from the YSO and the average line of sight velocity of the features of the two maser species. In both cores G24 A1 and G24 A2, the YSO position is assumed to coincide with the peak of the continuum emission observed with the VLA in the $1.3 \mathrm{~cm}$ band (denoted by the star symbol in Figs. 5 and 6 ). To avoid that the comparison of positions and velocities of the two maser emissions can be biased by insufficient sensitivity in detecting the 


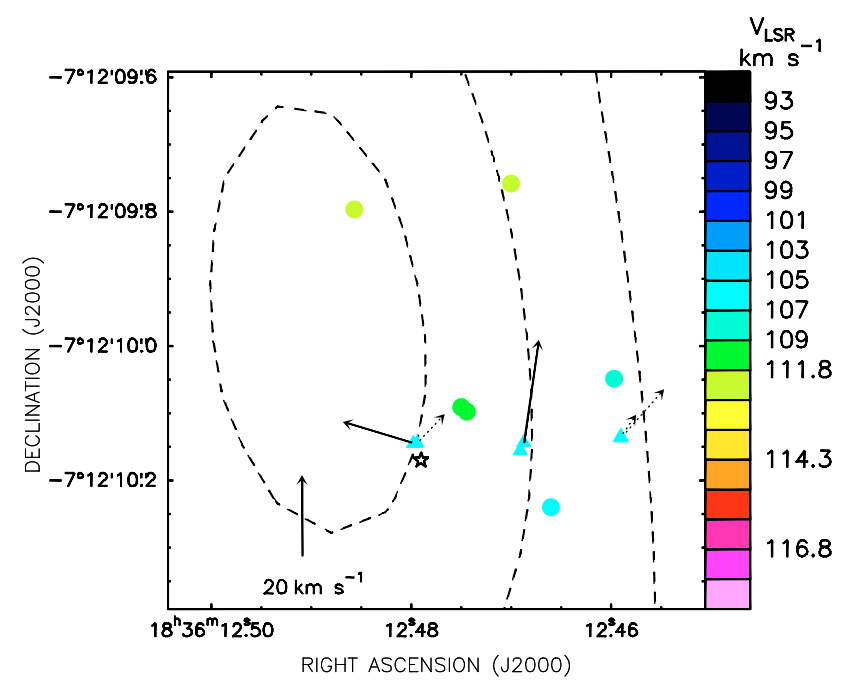

Fig. 6. In the $\mathrm{mm}$ subcore $\mathrm{G} 24 \mathrm{~A} 2$, the distribution of the VLBA 22.2 GHz water (triangles) and EVN 6.7 GHz methanol (dots) maser features is shown overlaid on the contour plot (dashed line) of the PdBI $1.4 \mathrm{~mm}$ emission observed by Beltrán et al. (2004). The colors denote different LSR velocities, according to the scale on the right-hand side of the panel. Black arrows show the measured absolute proper motions of water maser features, using dotted arrows to denote the most uncertain measurements; the amplitude scale for proper motions is reported at the bottom of the panel. The star symbol indicates the position of the G24 A2 continuum source detected with the VLA-B array in the $1.3 \mathrm{~cm}$ band.

weaker methanol maser emission, we restrict the examination to the region around the YSO where both types of masers are effectively detected. Towards G24 A1, this region corresponds to the viewing angle from the YSO between the two directions at PA ( $\mathrm{N}$ to $\mathrm{E})-40^{\circ}$ and $90^{\circ}$, and includes the three methanol maser spots at the northeastern extreme of the G24 A1 linear, methanol maser distribution, and all the water maser features falling within the ellipse of Fig. 5. Selecting only these features for the two maser types, the error-weighted $(1 / \sigma)$ average separation from the (putative) position of the YSO is 0 .' $^{\prime} 057 \pm 00^{\prime} 018$ and $0 . ' 101 \pm 0$. '003 for water and methanol masers, respectively. The $\mathrm{H}_{2} \mathrm{O}$ maser features are on average significantly closer to the YSO than the $\mathrm{CH}_{3} \mathrm{OH}$ maser features. The average line of sight velocity of the two maser species is only marginally different, with water features showing an average velocity, $112.1 \pm$ $3.8 \mathrm{~km} \mathrm{~s}^{-1}$, slightly lower than that of methanol masers, $114.0 \pm$ $0.6 \mathrm{~km} \mathrm{~s}^{-1}$. Towards the core G24 A2, the few detected spots of each of the two maser species distribute around the YSO over a comparable range of position angles, so that we can include all the detected, water and methanol, maser spots in the comparison. The average separation from the YSO position of the water, $0{ }^{\prime} 19 \pm 0.11$, and methanol, $0.23 \pm 0 . ' 11$, features is found to be the same within the sample spread. The average line of sight velocity of water masers, $106.1 \pm 0.5 \mathrm{~km} \mathrm{~s}^{-1}$, is slightly lower than that of methanol masers, $109.8 \pm 2.0 \mathrm{~km} \mathrm{~s}^{-1}$. While towards G24 A2 our results might be biased by poor statistics, owing to the small number of features detected in both maser species, towards G24 A1, with a statistically relevant number (76) of detected water features, we find that the average distance from the YSO of the two maser types does differ significantly. That appears to support our conjecture that water and methanol masers may trace different physical conditions around the $\mathrm{YSO}(\mathrm{s})$. In the next section, comparing maser VLBI with continuum and line interferometric observations, we propose a scenario for explaining the origin of water and methanol masers in the best studied core G24 A1. In AFGL 5142, to explain differences in the line of sight velocity emission range of the two maser species, Goddi et al. (2007) propose that, whereas $\mathrm{H}_{2} \mathrm{O}$ masers originate at the base of a protostellar wind, $\mathrm{CH}_{3} \mathrm{OH}$ masers are tracing infalling rather than expanding gas.

\section{Rotation and expansion in the $\mathrm{mm}$ core G24 A}

In the following we compare the VLBI $\mathrm{CH}_{3} \mathrm{OH}$ and $\mathrm{H}_{2} \mathrm{O}$ maser observations towards core $\mathrm{G} 24$ A with previous millimeter PdBI (Beltrán et al. 2004; Beltrán et al. 2005) and recent VLA (Beltrán et al. 2006; Beltrán et al. 2007) observations of this region, and discuss a model to explain the gas kinematics from small (100 AU) to large $(0.1 \mathrm{pc})$ scales.

\subsection{Evidence for rotation}

Figure 2 shows that, in both cores G24 A1 and A2, the LSR velocity distribution of methanol $6.7 \mathrm{GHz}$ maser spots agrees well with the velocity gradient measured in the thermal $\mathrm{CH}_{3} \mathrm{CN}$ (12-11) line. If, as suggested by Beltrán et al. (2004), the $\mathrm{CH}_{3} \mathrm{CN}$ velocity gradient is due to rotation, the 3 times larger spread of LSR velocities of the methanol masers across a 3 times smaller region, might be explained in terms of conservation of angular momentum from the large to the small scale inside the cores. Assuming that inside the region (of diameter $\approx 0.02 \mathrm{pc}$ ) traced by the $6.7 \mathrm{GHz}$ maser emission in both cores, the gas is in rotational equilibrium, one derives a dynamical mass of 55 and $19 M_{\odot}$ for G24 A1 and G24 A2, respectively. From the free-free continuum emission of the UCHII region in G24 A1, Codella et al. (1997) estimate a spectral type O9.5 for the exciting star, corresponding to a stellar mass of $\approx 20 M_{\odot}$. This must be summed to the mass of the gas within $0.02 \mathrm{pc}$ from the YSO, which is $\approx 30 M_{\odot}$ for a mean density of $10^{8} \mathrm{~cm}^{-3}$. The total mass (star plus gas) is consistent with the $55 M_{\odot}$ computed from the methanol maser velocity spread in G24 A1.

Figure 3 shows that the separation $(\approx 1$."5) and the orientation (along the SE-NW direction) of the two groups of $\mathrm{OH}$ $1.6 \mathrm{GHz}$ spots we have detected with VLBA is similar to that of the two clusters of $\mathrm{CH}_{3} \mathrm{OH} 6.7 \mathrm{GHz}$ masers observed in cores G24 A1 and A2 (see Fig. 2). Besides, as observed for the two methanol maser clusters, both $\mathrm{OH}$ maser groups are elongated along a SW-NE direction, and spots towards SW have blueshifted velocities, while those towards NE are redshifted. These similarities in the spatial and LSR velocity distribution lead us to suggest that the two groups of $\mathrm{OH}$ and $\mathrm{CH}_{3} \mathrm{OH}$ masers trace the same structures, possibly two flattened, rotating toroids. The birthplace of $\mathrm{OH} 1.6 \mathrm{GHz}$ and $\mathrm{CH}_{3} \mathrm{OH} 6.7 \mathrm{GHz}$ masers in cores G24 A1 and A2 might be similar as in the case of the massive (proto-)star IRAS 20126+4104, where both maser species emit at positions close to the (proto-)star and present a flattened distribution perpendicular to the axis of the (proto-)stellar outflow (Edris et al. 2005; Moscadelli et al. 2005).

\subsection{Evidence for expansion}

The water maser proper motions measured in the "A1N" subcluster are directed approximately perpendicular to the geometrical distribution of the maser features, which are mainly concentrated in two structures, a line oriented along the EW direction and an arc (see Fig. 5). The line of sight velocity components of most maser features of the "A1N" subcluster are quite small 
( $\leq 5 \mathrm{~km} \mathrm{~s}^{-1}$ ) compared with the sky-projected velocity components (20-60 $\mathrm{km} \mathrm{s}^{-1}$ ), implying that the velocity vectors of the maser features are close to the plane of the sky. The observed velocity distribution suggests that "A1N" water masers are tracing gas expanding towards N-NE.

The ellipse drawn in Fig. 5 denotes the position and the deconvolved $F W H M$ size of the continuum source in the G24 A1 core as recently determined using the VLA in the $1.3 \mathrm{~cm}$ and $7 \mathrm{~mm}$ bands by Beltrán et al. (2007). The position of the continuum peak at $22.2 \mathrm{GHz}$ is determined with an accuracy of $\approx 20$ mas. With an angular resolution of $\approx 0$ ' 05 , the continuum source is partially resolved with a deconvolved radius (defined as the geometric mean of the FWHM major and minor axes) of 0.07 (540 AU at a distance of $7.7 \mathrm{kpc}$ ). These recent VLA observations confirm that the most likely interpretation for the continuum source is in terms of a hyper-compact $\left(R_{\mathrm{HII}} \leq 0.01 \mathrm{pc}\right)$ HII region, optically thick at frequencies below $22.2 \mathrm{GHz}$, and optically thin between $22.2 \mathrm{GHz}$ and $44 \mathrm{GHz}$. The observed continuum flux is consistent with previous observations, indicating a ZAMS spectral type O9.5 for the central ionizing star.

Figure 5 evidences that the hyper-compact HII region falls close to the "A1N" maser subcluster. Considering that the estimated size of the HII region is likely an upper limit, one finds that more than $80 \%$ of the "A1N" water maser features may be distributed along the NE border of the ionized gas. The nondetection of water masers towards the SW border of the continuum source is likely due to the presence of a density gradient in this direction, as strongly indicated by the continuum source morphology, presenting a tail of emission towards SW (Beltrán et al. 2007). Figure 5 also shows that the elongation of the continuum source $\left(\mathrm{PA}=66^{\circ}\right)$ agrees well with the average orientation of the water maser proper motions. Thus, the position and the geometry of the continuum source suggests that the motion of the water masers can be driven by the expansion of the ionized gas.

Free expanding ionized gas should move at a maximum velocity comparable to the sound speed in the ionized gas of $\approx 10 \mathrm{~km} \mathrm{~s}^{-1}$, assuming a kinetic temperature of $10^{4} \mathrm{~K}$. The observed velocities of the water masers are significantly higher than $10 \mathrm{~km} \mathrm{~s}^{-1}$, indicating that the ionized gas is not freely expanding, but that the expansion must be driven by some additional mechanism than thermal pressure. Indicating with $V_{\mathrm{s}}$ the velocity of the high density, shocked material traced by the water masers, and with $\rho_{\mathrm{s}}$ the volume density of the surrounding medium through which the shock expands, the momentum flux, $\Phi_{\mathrm{s}}$, acquired by the shock-swept ambient gas is given by $\Phi_{\mathrm{s}}=\rho_{\mathrm{s}} V_{\mathrm{s}}^{2}$. Maser excitation models (Elitzur et al. 1989; Kaufman \& Neufeld 1996) require the pre-shock ambient gas to have numerical densities $n_{\mathrm{H}_{2}}>10^{6} \mathrm{~cm}^{-3}$. Using this value and the observed mean value of the water maser proper motions $V_{\mathrm{s}}=40 \mathrm{~km} \mathrm{~s}^{-1}$, one obtains $\Phi_{\mathrm{s}}>5.3 \times 10^{-5} \mathrm{erg} \mathrm{cm}^{-3}$.

The water masers could have been accelerated by stellar radiation pressure. However, assuming momentum conservation, one can see that the radiation pressure, $\Phi_{\mathrm{rad}}$, exerted by the YSO in the G24 A1 core is too weak to sustain expansion at $\approx 40 \mathrm{~km} \mathrm{~s}^{-1}$ up to a distance of $\approx 540 \mathrm{AU}$. Indicating with $R_{\mathrm{s}}$ the radius of the expanding shell, one can calculate $\Phi_{\text {rad }}$ from the expression $\Phi_{\mathrm{rad}}=L_{\star} /\left(c 4 \pi R_{\mathrm{s}}^{2}\right)$, where $L_{\star}=7 \times 10^{4} L_{\odot}$ is the measured bolometric luminosity of the G24 A core and $c$ is the speed of light. The result is $\Phi_{\text {rad }}=10^{-5} \mathrm{erg} \mathrm{cm}^{-3}$. This value is a factor $\approx 5$ smaller than the derived lower limit for the momentum flux required to accelerate the water masers.

Stellar winds are commonly observed towards early type OB main-sequence stars and observational evidence is being accumulated that such winds play an important role during the early expansion phase of an hyper-compact HII region (Hoare et al. 2007). A detailed study by Lamers \& Leitherer (1993) indicates wind velocities $V_{\mathrm{w}}=1500-2500 \mathrm{~km} \mathrm{~s}^{-1}$ and mass loss rates $\dot{M}_{\mathrm{w}}=1-5 \times 10^{-6} M_{\odot} \mathrm{yr}^{-1}$, as appropriate for an $09.5 \mathrm{star}$. Such a powerful stellar wind might be already emitted during the early ZAMS evolution of a massive star. In the following we discuss the case that the expansion of the water maser shell is driven by a strong wind. Our VLBA water maser and VLA continuum radio observations allow us to set a value for the shell radius and velocity: $R_{0} \approx 500 \mathrm{AU}, V_{0} \approx 40 \mathrm{~km} \mathrm{~s}^{-1}$. Thus, we can look for a solution of the wind-driven expansion which reproduces the observed shell parameters.

Appendices $\mathrm{A}$ and $\mathrm{B}$ give the equations for the motion of the wind-driven shell (Castor et al. 1975; Shull 1980) and present the results of our calculations. We find that, over the explored parameter space for the stellar wind properties, fixing the wind mechanical luminosity, it does exist a unique value of ambient density for which the wind-driven shell can attain the observed values of radius and velocity. For the likely values of wind luminosities for a 09.5 star, the wind-driven shell models require the $\mathrm{H}$ particle ambient density to lay in the range $6 \times 10^{6} \mathrm{~cm}^{-3} \leq$ $n_{\mathrm{H}} \leq 2.5 \times 10^{7} \mathrm{~cm}^{-3}$. These density values for the ambient preshock gas are perfectly compatible with the predictions of the water maser excitation models (Elitzur et al. 1989; Kaufman $\&$ Neufeld 1996). The shell age, $t_{0}$, is predicted to vary in the narrow interval $t_{0}=30-35 \mathrm{yr}$. Note that the derived shell age does not depend on the adopted distance of G24.78+0.08, since $t_{0} \sim R_{0} V_{0}^{-1}$ (see Appendix B, Eqs. (B.7) and (B.21)), and both $R_{0}$ and $V_{0}$ are proportional to the source distance. Even assuming that the adopted values of $R_{0}$ and $V_{0}$ are wrong by $50 \%$, winddriven solutions allow for a maximum value $t_{0} \approx 80 \mathrm{yr}$. This corresponds to a small, but not implausibly small, probability to observe this particular expansion phase of the wind-driven shell.

The wind-driven shell model predicts that the ionized gas is confined in a thin, dense shell surrounding the expanding wind bubble. The calculations of Shull (1980) show that the radio appearance (flux, angular diameter, spectral index) of a winddriven shell is very similar to that of a compact HII region, in agreement with our findings (Beltrán et al. 2007). In the starforming region $\mathrm{W} 75 \mathrm{~N}$, using the VLA to observe the $1.3 \mathrm{~cm}$ continuum and the VLBA to measure the proper motion of the 22.2 GHz water masers, Torrelles et al. (2003) detected a compact continuum source (VLA 2) surrounded by a small ring (radius of $160 \mathrm{AU}$ ) of water maser spots expanding (with an average velocity of $28 \mathrm{~km} \mathrm{~s}^{-1}$ ) outward from the center of the radio continuum source. The way water masers distribute and move with respect to the compact continuum source presents similarities with what observed towards the massive YSO in the G24 A1 core. A major difference is in the radio continuum flux, which towards W75 N VLA 2 is much weaker $(\sim 1 \mathrm{mJy}$, at a distance of $2 \mathrm{kpc})$ than towards G24 A1 ( $\approx 80 \mathrm{mJy}$, at a distance of $7.7 \mathrm{kpc}$ ). Assuming that an ionized wind is responsible for the radio continuum and drives the expansion of the water masers, Torrelles et al. (2003) obtain a small age of $13 \mathrm{yr}$ for the W75 N VLA 2 source. Since it is unlikely to observe such short-lived phenomena, these authors speculate that the ejection of wideangle winds might occur several times during the early phases of a massive (proto-)star. We believe that this cannot be the case of G24 A1, because the relatively high intensity and the spectral properties of the radio continuum emission indicate that the star has already reached the ZAMS. 


\subsection{Rotation or expansion in the G24 A1 core?}

The most striking result of the methanol and water maser study of the G24 A1 core is that the two maser species appear to trace different velocity fields, the former being associated with rotation, the latter with expansion. While the $\mathrm{CH}_{3} \mathrm{OH}$ maser motion is consistent with the toroid/outflow model depicted by Beltrán et al. (2005), the detection of $\mathrm{H}_{2} \mathrm{O}$ maser expansion along the plane of the toroid seems to contradict that model. A discussion of this issue is in order. In particular, one must explain at the same time all the following observational facts: (i) the presence of a velocity gradient along the NE-SW direction, measured in $\mathrm{CH}_{3} \mathrm{CN}$ over $\sim 0.6$ pc (Beltrán et al. 2004; Beltrán et al. 2005) and in the $\mathrm{CH}_{3} \mathrm{OH}$ masers over $\sim 0.02 \mathrm{pc}$; (ii) the $\mathrm{CO}$ bipolar outflow oriented SE-NW (Furuya et al. 2002); (iii) the infall towards the hypercompact HII region, detected in the $\mathrm{NH}_{3}$ line (Beltrán et al. 2006); (iv) the expansion traced by the $\mathrm{H}_{2} \mathrm{O}$ masers at the surface of the hypercompact HII region (see Sect. 5.2).

Below, we propose two alternative interpretations.

\subsubsection{1st scenario: $\mathrm{CH}_{3} \mathrm{CN}$ outflow}

The velocity gradient observed in the thermal $\mathrm{CH}_{3} \mathrm{CN}$ (12-11) line as well as in the $\mathrm{CH}_{3} \mathrm{OH} 6.7 \mathrm{GHz}$ maser emission (see Fig. 2), might be due to expansion rather than rotation as proposed by Beltrán et al. (2004). This would imply the existence of a collimated, compact (i.e. young) bipolar outflow, perpendicular to the outflow seen on a larger scale in the $\mathrm{CO}(1-0)$ line, which might be powered by another YSO in the same cluster. In this scenario, the $22.2 \mathrm{GHz}$ water masers would mark the innermost part of the outflow, very close (a few $100 \mathrm{AU}$ ) to the star, while the $\mathrm{CH}_{3} \mathrm{OH} 6.7 \mathrm{GHz}$ masers and the $\mathrm{CH}_{3} \mathrm{CN}$ thermal emissions would trace the same outflow on larger scales.

The fact that the $\mathrm{CH}_{3} \mathrm{CN}$ velocity field is very well defined (see Fig. 2a) proves that the corresponding outflow should be very collimated, which in turn strongly suggests the existence of a small $(\$ 0.02 \mathrm{pc})$, yet undetected circumstellar disk oriented SE-NW. This would be also consistent with the detection of infall: the material could accrete onto the star passing through the disk plane and even through the hypercompact HII region, as predicted by recent models (Keto \& Wood 2006). This implies that the star could be still growing in mass and is hence to be seen as a massive proto-star.

\subsubsection{2nd scenario: $\mathrm{CH}_{3} \mathrm{CN}$ toroid}

The alternative scenario is that $\mathrm{CH}_{3} \mathrm{CN}$ and $\mathrm{CH}_{3} \mathrm{OH}$ are tracing a rotating toroid, and $\mathrm{CO}$ the associated bipolar outflow. In this context, the infall revealed by Beltrán et al. (2006) would proceed through the plane of the toroid, which appears to conflict with the outward motion observed along the same direction in the $\mathrm{H}_{2} \mathrm{O}$ maser spots. In fact, if such a motion is lead by the HII region expansion (as discussed in Sect. 5.2), then in no way the accreting material can reach the star, but is halted at the shock front at the surface of the HII region. This means that the accretion phase is over and the star has reached its final mass.

In this model, the water masers would participate to the expansion in a post-shock layer, whereas the methanol $6 \mathrm{GHz}$ masers would trace the pre-shock material, whose kinematics is dominated by rotation. This hypothesis is supported by the fact that the $\mathrm{CH}_{3} \mathrm{OH}$ masers lie further from the HII region (i.e. from the star) than the $\mathrm{H}_{2} \mathrm{O}$ masers (see Fig. 5). One can thus predict that the $\mathrm{CH}_{3} \mathrm{OH}$ maser proper motions should be completely different from those of the $\mathrm{H}_{2} \mathrm{O}$ masers, tracing inward motions towards the star, rather than expansion from it.

This prediction can be tested with VLBI multi-epoch measurements of the $6 \mathrm{GHz} \mathrm{CH}_{3} \mathrm{OH}$ maser spots. In this way one will be able to discriminate between the two scenarios proposed above and thus establish whether the toroid model depicted by Beltrán et al. (2004, 2005, 2006) is correct.

\section{The outflow in the G24 C mm core}

Towards the $\mathrm{G} 24 \mathrm{C} \mathrm{mm}$ core, no radio continuum emission in the $1.3 \mathrm{~cm}$ and $7 \mathrm{~mm}$ bands has been detected by recent sensitive $^{4}$ VLA observations (Beltrán et al. 2007), which indicates that either the YSO has a spectral type later than B2 or it is in a early evolutionary stage prior to the appearance of an HII region. The low gas temperatures of $\approx 30 \mathrm{~K}$ derived using the $\mathrm{NH}_{3}$ line (Codella et al. 1997) may explain the non-detection of the Class II $6.7 \mathrm{GHz}$ masers in this core, since excitation models predict these masers to be radiatively excited and reach high brightness temperatures for kinetic temperatures of $\approx 150 \mathrm{~K}$ (Cragg et al. 2005). The detection of the Class I $44 \mathrm{GHz}$ and $95 \mathrm{GHz}$ maser clusters in correspondence of the peak of the $\mathrm{CS}(3-2)$ map and roughly at the center of the ${ }^{12} \mathrm{CO}(1-0)$ bipolar outflow (see Fig. 1), is consistent with the idea that the Class I methanol masers are collisionally excited in shocks propagating through dense ambient gas.

In the water maser subclusters "C1" and "C3" (see panel "C" of Fig. 4 and Table 6), maser features have transverse velocities of $\approx 100-200 \mathrm{~km} \mathrm{~s}^{-1}$ and move nearly along the same direction. This is expected if these water masers trace the collimated portion of a high-velocity outflow. One might speculate that the driving source of the outflow is located close to the center of the "C2" subcluster, because the maser features in this subcluster display both blue- and redshifted LSR-velocities, and the measured proper motions appear to diverge from a common origin. Based on the maser spatial and velocity distribution, the axis of this putative outflow might coincide with the line passing through the center of the compact " $\mathrm{C} 2$ " subcluster and oriented along the average direction (at $\mathrm{PA} \approx 200^{\circ}$ ) of the nearly parallel proper motions observed in the subclusters " $\mathrm{C} 1$ " and "C3". Recent studies on high-mass outflows indicate that they could be driven by similar physical processes as those seen in low-mass outflows (Beuther \& Shepherd 2005). The water maser motions observed in the G24 C mm core are significantly faster and more collimated than those in G24 A1, which might favor the interpretation that the YSO in G24 C is in an evolutionary phase earlier than the ZAMS, since the fastest and most collimated flows are generally observed during the earliest evolution stages (Beuther \& Shepherd 2005; Bontemps et al. 1996).

Albeit appealing the outflow interpretation depicted above remains highly speculative. In fact, no indication for a YSO at the location of the "C2" maser cluster is known to date, while evidence for a bipolar outflow is given by Furuya et al. (2002) but oriented perpendicular to the putative maser outflow. The latter instead would be approximately parallel to the direction of the velocity gradient observed in the CS(3-2) line, interpreted in terms of rotation by Beltrán et al. (2004). However, looking at Fig. 2 of Beltrán et al. (2004), one notes that the velocity gradient in the $\mathrm{G} 24 \mathrm{C}$ core is less prominent than in the G24 A1 and A2 cores observed in the $\mathrm{CH}_{3} \mathrm{CN}$ emission, and it cannot be excluded that the trend of LSR velocity of the G24 C core is caused

\footnotetext{
4 the channel map rms noise was $0.09 \mathrm{mJy}$ beam $^{-1}$ and $0.27 \mathrm{mJy} \mathrm{beam}^{-1}$ at $1.3 \mathrm{~cm}$ and $7 \mathrm{~mm}$, respectively.
} 
by outflowing motions (oriented approximately NE-SW) instead of rotation. Moreover one cannot rule out the coexistence on scales of a few arcseconds of multiple outflows with different directions and/or degrees of collimation, as proved by interferometric observations of high-mass SFRs (Beuther et al. 2003, 2004).

In conclusion, even if the high-velocity and collimated motions of the water masers observed in the G24 C mm core suggest the outflow interpretation, this needs still to be put on firm ground by further interferometric observations of outflow/jet tracers such as e.g. $\mathrm{SiO}$ or $\mathrm{HCO}^{+}$.

\section{Conclusions and future work}

Towards the high-mass star-forming region $\mathrm{G} 24.78+0.08$, we have observed several maser species: $\mathrm{OH} 1665 \mathrm{MHz}$ masers (using VLBA), $\mathrm{H}_{2} \mathrm{O} 22.2 \mathrm{GHz}$ masers (both with VLA and VLBA), $\mathrm{CH}_{3} \mathrm{OH}$ Class II $6.7 \mathrm{GHz}$ masers (with EVN), $\mathrm{CH}_{3} \mathrm{OH}$ Class I 44 and $95 \mathrm{GHz}$ masers (using respectively VLA and BIMA). We have compared positions and velocities of the different maser species with interferometric PdBI observations of thermal lines, tracing the velocity field of high-density gas, and with recent sensitive VLA observations of the thermal continuum emission.

In the $\mathrm{G} 24 \mathrm{~A} 1$ subcore, the $\mathrm{CH}_{3} \mathrm{OH}$ 6.7 $\mathrm{GHz}$ and $\mathrm{H}_{2} \mathrm{O}$ 22.2 $\mathrm{GHz}$ masers appear to be associated to the same $\mathrm{YSO}(\mathrm{s})$, even if it is likely that the two maser species originate in different environments. $22.2 \mathrm{GHz}$ masers trace a fast $\left(\sim 40 \mathrm{~km} \mathrm{~s}^{-1}\right)$ expanding shell surrounding the hyper-compact HII region at the center of the G24 A1 subcore, and we suggest that their motion is driven by a strong wind emitted by the ZAMS O9.5 type star responsible for the formation of the hyper-compact HII region. $\mathrm{CH}_{3} \mathrm{OH}$ 6.7 GHz maser features have larger (sky-projected) distances from the continuum emission peak than the water masers, and their flattened spatial distribution together with their regular variation in LSR velocities lead us to propose that they are emerging in a rotating toroid surrounding the hyper-compact HII region. Alternatively, the regular trend of LSR velocities observed both in the $6.7 \mathrm{GHz}$ maser and in thermal line emission might result from a collimated flow of gas at scales of $\approx 0.1 \mathrm{pc}$, which might be the collimated portion of the same outward motion traced by the water masers at smaller scales.

The proposed models can be directly tested by measuring the proper motions of the $\mathrm{CH}_{3} \mathrm{OH}$ masers. If, for instance, one finds that the $6.7 \mathrm{GHz}$ masers move as fast as water masers do, the outflow model would be supported; in the case that the $6.7 \mathrm{GHz}$ proper motions are much smaller than those of the water masers and compatible with Keplerian rotation around $\mathrm{a} \approx 20 M_{\odot}$ star, that would fit with the rotating toroid model. In March 2007, using EVN, we have observed again G24.78+0.08 in the $6.7 \mathrm{GHz}$ maser emission. Since the time separation of these new observations from the June 2003 EVN run (reported in this paper) is long enough to detect proper motions of amplitude $\approx 10 \mathrm{~km} \mathrm{~s}^{-1}$, these two epochs might already suffice in establishing if methanol masers move with high velocities or do not.

In the $\mathrm{G} 24 \mathrm{C}$ subcore, many of the detected water maser features move fast (at velocities of $\sim 100-200 \mathrm{~km} \mathrm{~s}^{-1}$ ) and along a common direction (at $\mathrm{PA} \approx 200^{\circ}$ ), which might suggest that the water maser emission traces a collimated outflow. We plan to perform sensitive, sub-arcsecond angular resolution observations of $\mathrm{G} 24 \mathrm{C}$ in typical outflow tracers (like $\mathrm{SiO}$ and $\mathrm{HCO}^{+}$) to inquire for the presence of (proto-)stellar outflows.

Acknowledgements. Dr. Maria T. Beltrán is suppported by MEC grant AYA2005-08523-C03

\section{References}

Beltrán, M. T., Cesaroni, R., Neri, R., et al. 2004, ApJ, 601, L187 Beltrán, M. T., Cesaroni, R., Neri, R., et al. 2005, A\&A, 435, 901 Beltrán, M. T., Cesaroni, R., Codella, C., et al. 2006, Nature, 443, 427 Beltrán, M., Cesaroni, R., Moscadelli, L., \& Codella, C. 2007, A\&A, 471, L13 Beuther, H., \& Shepherd, D. 2005, in Cores to Clusters: Star Formation with Next Generation Telescopes, ed. M. S. N. Kumar, M. Tafalla, \& P. Caselli, 105

Beuther, H., Schilke, P., \& Stanke, T. 2003, A\&A, 408, 601

Beuther, H., Schilke, P., \& Gueth, F. 2004, ApJ, 608, 330

Bonnell, I. A., \& Bate, M. R. 2002, MNRAS, 336, 659

Bontemps, S., Andre, P., Terebey, S., \& Cabrit, S. 1996, A\&A, 311, 858

Castor, J., McCray, R., \& Weaver, R. 1975, ApJ, 200, L107

Cesaroni, R. 2005, Ap\&SS, 295, 5

Cesaroni, R., Codella, C., Furuya, R. S., \& Testi, L. 2003, A\&A, 401, 227

Codella, C., Testi, L., \& Cesaroni, R. 1997, A\&A, 325, 282

Cragg, D. M., Sobolev, A. M., \& Godfrey, P. D. 2005, MNRAS, 360, 533

De Buizer, J. M. 2003, MNRAS, 341, 277

Edris, K. A., Fuller, G. A., Cohen, R. J., \& Etoka, S. 2005, A\&A, 434, 213

Elitzur, M., Hollenbach, D. J., \& McKee, C. F. 1989, ApJ, 346, 983

Forster, J. R., \& Caswell, J. L. 1989, A\&A, 213, 339

Furuya, R. S., Cesaroni, R., Codella, C., et al. 2002, A\&A, 390, L1

Goddi, C., \& Moscadelli, L. 2006, A\&A, 447, 577

Goddi, C., Moscadelli, L., Alef, W., et al. 2005, A\&A, 432, 161

Goddi, C., Moscadelli, L., Sanna, A., Cesaroni, R., \& Minier, V. 2007, A\&A, 461,1027

Hoare, M. G., Kurtz, S. E., Lizano, S., Keto, E., \& Hofner, P. 2007, in Protostars and Planets V, ed. B. Reipurth, D. Jewitt, \& K. Keil, 181

Kaufman, M. J., \& Neufeld, D. A. 1996, ApJ, 456, 250

Keto, E., \& Wood, K. 2006, ApJ, 637, 850

Lamers, H. J. G. L. M., \& Leitherer, C. 1993, ApJ, 412, 771

Moscadelli, L., Cesaroni, R., \& Rioja, M. J. 2005, A\&A, 438, 889

Moscadelli, L., Testi, L., Furuya, R. S., et al. 2006, A\&A, 446, 985

Norris, R. P., Byleveld, S. E., Diamond, P. J., et al. 1998, ApJ, 508, 275

Palla, F., \& Stahler, S. W. 1993, ApJ, 418, 414

Sault, R. J., Teuben, P. J., \& Wright, M. C. H. 1995, in Astronomical Data Analysis Software and Systems IV, ed. R. A. Shaw, H. E. Payne, \& J. J. E. Hayes, ASP Conf. Ser., 77, 433

Shull, J. M. 1980, ApJ, 238, 860

Torrelles, J. M., Patel, N. A., Anglada, G., et al. 2003, ApJ, 598, L115

Walsh, A. J., Burton, M. G., Hyland, A. R., \& Robinson, G. 1998, MNRAS, 301, 640

Yorke, H. W., \& Sonnhalter, C. 2002, ApJ, 569, 846 


\section{Online Material}


Table 1. Parameters of Class I $\mathrm{CH}_{3} \mathrm{OH}$ maser features detected with the VLA $(44 \mathrm{GHz})$ and BIMA $(95 \mathrm{GHz})$. Class I $\mathrm{CH}_{3} \mathrm{OH}$ masers are orientatively divided in two groups on the basis of their proximity to either molecular core A or C. For each identified feature, Col. 1 gives the harboring core, Col. 2 reports the observing frequency; Cols. 3 and 4 the LSR velocity and the integrated flux density of the highest-intensity channel; Cols. 5 and 6 the positional (RA and Dec) coordinates.

\begin{tabular}{cccccc}
\hline Core & $\begin{array}{c}v \\
(\mathrm{GHz})\end{array}$ & $\begin{array}{c}V_{\mathrm{LSR}} \\
\left(\mathrm{km} \mathrm{s}^{-1}\right)\end{array}$ & $\begin{array}{c}F_{\text {int }} \\
(\mathrm{Jy})\end{array}$ & $\begin{array}{c}\alpha(\mathrm{J} 2000) \\
(\mathrm{h} \mathrm{m} \mathrm{s})\end{array}$ & $\begin{array}{c}\delta(\mathrm{J} 2000) \\
\left({ }^{\circ}{ }^{\prime \prime}\right)\end{array}$ \\
\hline Core A & 44 & 108.4 & 9.5 & 183612.62 & -071210.5 \\
& 44 & 109.8 & 0.6 & 183612.66 & -071211.2 \\
& 44 & 111.3 & 1.9 & 183612.46 & -071205.8 \\
& 44 & 111.5 & 1.3 & 183612.18 & -071208.8 \\
& 44 & 108.5 & 1.2 & 183612.54 & -071209.6 \\
& 44 & 111.0 & 0.4 & 183611.99 & -071204.8 \\
& 44 & 112.8 & 0.2 & 183612.67 & -071212.3 \\
& 44 & 110.9 & 0.2 & 183612.70 & -071211.2 \\
& 44 & 113.6 & 0.1 & 183612.63 & -071211.7 \\
& 44 & 111.2 & 0.1 & 183612.97 & -071211.4 \\
& 44 & 109.1 & 0.08 & 183612.27 & -071209.3 \\
& 44 & 109.1 & 0.08 & 183612.18 & -071207.7 \\
& 44 & 110.5 & 0.08 & 183612.66 & -071223.9 \\
& 44 & 108.6 & 4.4 & 183612.57 & -071218.8 \\
& 44 & 110.7 & 0.2 & 183612.21 & -071220.9 \\
& 95.2 & 107.5 & 0.3 & 183612.54 & -071209.5 \\
& 95.2 & 114.6 & 3.5 & 183612.62 & -071210.4 \\
& 44 & 115.3 & 34.0 & 183612.85 & -071200.4 \\
& 44 & 114.3 & 14.4 & 183613.04 & -071207.1 \\
& 44 & 115.0 & 1.0 & 183613.09 & -071206.9 \\
& 44 & 112.6 & 4.6 & 183613.09 & -071207.8 \\
& 44 & 111.0 & 2.2 & 183613.15 & -071208.5 \\
& 44 & 111.3 & 1.9 & 183613.17 & -071206.9 \\
& 44 & 111.7 & 1.1 & 183613.74 & -071208.1 \\
& 44 & 111.7 & 0.4 & 183613.70 & -071207.8 \\
& 44 & 113.8 & 0.5 & 183613.08 & -071205.3 \\
& 44 & 112.5 & 0.4 & 183613.06 & -071206.0 \\
& 44 & 111.6 & 0.5 & 183613.16 & -071205.7 \\
& 44 & 110.3 & 0.4 & 183612.65 & -071158.9 \\
& 44 & 110.5 & 0.4 & 183612.66 & -071159.8 \\
& 44 & 117.6 & 0.4 & 183612.79 & -071200.3 \\
& 44 & 116.1 & 0.2 & 183612.78 & -071159.7 \\
& 44 & 114.2 & 0.2 & 183612.94 & -071206.5 \\
& 44 & 112.4 & 0.2 & 183612.61 & -071159.4 \\
& 44 & 110.6 & 0.1 & 183612.61 & -071159.7 \\
& 44 & 113.7 & 0.2 & 183613.00 & -071206.4 \\
& 44 & 120.1 & 0.09 & 183612.75 & -071200.3 \\
& 44 & 106.4 & 0.09 & 183613.18 & -071210.6 \\
& 95.2 & 110.7 & 0.06 & 183612.89 & -071207.1 \\
& 107.8 & 1.3 & 183612.85 & -071200.2 \\
& 113.9 & 1.5 & 183613.04 & -071207.0 \\
& 112.4 & 0.8 & 183613.09 & -071207.6 \\
& 110.6 & 0.2 & 183613.15 & -071208.4 \\
& 110.6 & 0.1 & 183613.17 & -071206.8 \\
\hline .2 & & & &
\end{tabular}


Table 5. Parameters of VLBA water maser features in core A. For each identified feature, Col. 1 indicates the harboring maser (sub) cluster; Cols. 2 and 3 the LSR velocity and the integrated flux density of the highest-intensity channel; Cols. 4 and 5 the positional (RA and Dec) offsets (with the associated errors), evaluated with respect to the derived absolute position of the reference feature $\left(\alpha(J 2000)=18^{\mathrm{h}} 36^{\mathrm{m}} 12^{\mathrm{s}} .5581\right.$, $\left.\delta(J 2000)=-7^{\circ} 12^{\prime} 10^{\prime} \cdot 792\right)$; Cols. 6-8 the projected components along the RA and Dec axes and the absolute value of the derived proper motions (together with the associated errors).

\begin{tabular}{|c|c|c|c|c|c|c|c|}
\hline Cluster & $\begin{array}{r}V_{\text {LSR }} \\
\left(\mathrm{km} \mathrm{s}^{-1}\right)\end{array}$ & $\begin{array}{l}F_{\text {int }} \\
(\mathrm{Jy})\end{array}$ & $\begin{array}{c}\Delta \alpha \\
\text { (mas) }\end{array}$ & $\begin{array}{c}\Delta \delta \\
(\mathrm{mas})\end{array}$ & $\begin{array}{c}V_{x} \\
\left(\mathrm{~km} \mathrm{~s}^{-1}\right)\end{array}$ & $\begin{array}{c}V_{y} \\
\left(\mathrm{~km} \mathrm{~s}^{-1}\right)\end{array}$ & $\begin{array}{c}V_{\text {mod }} \\
\left(\mathrm{km} \mathrm{s}^{-1}\right)\end{array}$ \\
\hline $\mathrm{A} 1 \mathrm{~N}$ & 112.3 & 94.4 & $0.0 \pm 0.2$ & $0.0 \pm 0.3$ & $26 \pm 9$ & $5 \pm 9$ & $26 \pm 9$ \\
\hline A1N & 112.4 & 58.6 & $30.6 \pm 0.2$ & $17.9 \pm 0.3$ & $42 \pm 11$ & $31 \pm 14$ & $53 \pm 12$ \\
\hline A1N & 110.8 & 54.7 & $32.8 \pm 0.2$ & $23.0 \pm 0.2$ & & & \\
\hline $\mathrm{A} 1 \mathrm{~N}$ & 112.9 & 44.9 & $28.8 \pm 0.2$ & $14.3 \pm 0.3$ & $2 \pm 10$ & $-20 \pm 13$ & $20 \pm 13$ \\
\hline A1N & 111.3 & 41.4 & $-49.9 \pm 0.2$ & $43.5 \pm 0.2$ & $4 \pm 10$ & $40 \pm 12$ & $41 \pm 12$ \\
\hline A1N & 110.5 & 21.5 & $32.0 \pm 0.2$ & $24.5 \pm 0.3$ & & & \\
\hline A1N & 112.9 & 15.2 & $24.2 \pm 0.2$ & $9.5 \pm 0.3$ & $23 \pm 11$ & $9 \pm 15$ & $25 \pm 11$ \\
\hline $\mathrm{A} 1 \mathrm{~N}$ & 111.1 & 14.5 & $-66.5 \pm 0.2$ & $50.2 \pm 0.2$ & $0 \pm 10$ & $40 \pm 12$ & $40 \pm 12$ \\
\hline A1N & 112.5 & 11.5 & $31.0 \pm 0.2$ & $18.0 \pm 0.2$ & & & \\
\hline A1N & 111.6 & 11.0 & $31.8 \pm 0.2$ & $22.6 \pm 0.3$ & & & \\
\hline $\mathrm{A} 1 \mathrm{~N}$ & 114.8 & 9.6 & $23.3 \pm 0.2$ & $6.6 \pm 0.3$ & $14 \pm 12$ & $-30 \pm 15$ & $33 \pm 14$ \\
\hline A1N & 110.1 & 9.4 & $-23.1 \pm 0.2$ & $46.7 \pm 0.2$ & $10 \pm 11$ & $44 \pm 13$ & $45 \pm 12$ \\
\hline A1N & 112.3 & 8.9 & $32.1 \pm 0.2$ & $30.1 \pm 0.5$ & & & \\
\hline $\mathrm{A} 1 \mathrm{~N}$ & 113.0 & 8.6 & $27.0 \pm 0.2$ & $13.6 \pm 0.2$ & $11 \pm 11$ & $-5 \pm 12$ & $12 \pm 11$ \\
\hline $\mathrm{A} 1 \mathrm{~N}$ & 112.4 & 8.3 & $32.5 \pm 0.2$ & $29.8 \pm 0.3$ & & & \\
\hline $\mathrm{A} 1 \mathrm{~N}$ & 112.5 & 7.8 & $31.1 \pm 0.2$ & $18.6 \pm 0.2$ & & & \\
\hline A1N & 113.2 & 7.3 & $30.1 \pm 0.2$ & $17.2 \pm 0.2$ & $23 \pm 11$ & $5 \pm 13$ & $24 \pm 11$ \\
\hline A1N & 118.1 & 6.9 & $31.8 \pm 0.2$ & $21.8 \pm 0.2$ & & & \\
\hline $\mathrm{A} 1 \mathrm{~N}$ & 113.0 & 6.7 & $26.3 \pm 0.2$ & $37.0 \pm 0.2$ & $14 \pm 10$ & $46 \pm 13$ & $48 \pm 13$ \\
\hline A1N & 114.4 & 6.6 & $33.5 \pm 0.2$ & $26.6 \pm 0.2$ & & & \\
\hline $\mathrm{A} 1 \mathrm{~N}$ & 116.3 & 6.6 & $30.4 \pm 0.2$ & $18.5 \pm 0.2$ & & & \\
\hline $\mathrm{A} 1 \mathrm{~N}$ & 111.0 & 6.5 & $1.0 \pm 0.2$ & $0.9 \pm 0.3$ & & & \\
\hline A1N & 111.5 & 4.7 & $32.4 \pm 0.2$ & $21.0 \pm 0.3$ & & & \\
\hline A1N & 111.8 & 4.6 & $31.9 \pm 0.2$ & $31.1 \pm 0.6$ & & & \\
\hline A1N & 115.8 & 4.2 & $31.1 \pm 0.3$ & $20.4 \pm 0.3$ & & & \\
\hline $\mathrm{A} 1 \mathrm{~N}$ & 111.4 & 4.2 & $32.9 \pm 0.2$ & $30.2 \pm 0.2$ & & & \\
\hline $\mathrm{A} 1 \mathrm{~N}$ & 114.6 & 3.9 & $31.9 \pm 0.2$ & $23.2 \pm 0.3$ & $25 \pm 22$ & $0 \pm 29$ & $25 \pm 22$ \\
\hline $\mathrm{A} 1 \mathrm{~N}$ & 115.1 & 3.6 & $31.2 \pm 0.2$ & $19.8 \pm 0.2$ & & & \\
\hline A1N & 114.4 & 3.5 & $30.3 \pm 0.2$ & $15.4 \pm 0.3$ & & & \\
\hline A1N & 113.9 & 3.5 & $-1.3 \pm 0.2$ & $-1.0 \pm 0.3$ & $70 \pm 18$ & $30 \pm 21$ & $76 \pm 18$ \\
\hline A1N & 112.5 & 3.5 & $-28.4 \pm 0.2$ & $45.5 \pm 0.3$ & $10 \pm 11$ & $44 \pm 13$ & $45 \pm 13$ \\
\hline A1N & 114.1 & 3.2 & $-132.1 \pm 0.2$ & $53.6 \pm 0.2$ & $-5 \pm 12$ & $29 \pm 12$ & $30 \pm 12$ \\
\hline A1N & 113.2 & 3.0 & $24.1 \pm 0.2$ & $8.6 \pm 0.2$ & & & \\
\hline A1N & 111.6 & 2.8 & $29.4 \pm 0.2$ & $34.8 \pm 0.2$ & $30 \pm 21$ & $-3 \pm 28$ & $31 \pm 21$ \\
\hline $\mathrm{A} 1 \mathrm{~N}$ & 113.2 & 2.6 & $31.4 \pm 0.2$ & $21.2 \pm 0.2$ & & & \\
\hline $\mathrm{A} 1 \mathrm{~N}$ & 110.6 & 2.6 & $66.2 \pm 0.2$ & $-113.9 \pm 0.3$ & $11 \pm 10$ & $31 \pm 13$ & $33 \pm 12$ \\
\hline $\mathrm{A} 1 \mathrm{~N}$ & 114.7 & 2.5 & $27.6 \pm 0.2$ & $36.6 \pm 0.2$ & & & \\
\hline A1N & 115.9 & 2.5 & $31.6 \pm 0.2$ & $21.9 \pm 0.3$ & & & \\
\hline A1N & 109.7 & 2.4 & $32.5 \pm 0.2$ & $27.7 \pm 0.4$ & & & \\
\hline A1N & 110.7 & 2.2 & $32.7 \pm 0.2$ & $28.4 \pm 0.3$ & & & \\
\hline A1N & 113.4 & 2.2 & $30.9 \pm 0.2$ & $34.0 \pm 0.3$ & & & \\
\hline A1N & 113.8 & 2.1 & $30.2 \pm 0.2$ & $15.5 \pm 0.3$ & $40 \pm 11$ & $-12 \pm 14$ & $42 \pm 11$ \\
\hline A1N & 109.0 & 2.1 & $-3.9 \pm 0.2$ & $6.1 \pm 0.3$ & $4 \pm 22$ & $18 \pm 29$ & $18 \pm 29$ \\
\hline $\mathrm{A} 1 \mathrm{~N}$ & 110.5 & 2.0 & $-63.1 \pm 0.2$ & $49.0 \pm 0.2$ & $13 \pm 11$ & $35 \pm 12$ & $38 \pm 12$ \\
\hline A1N & 113.9 & 1.9 & $-123.8 \pm 0.2$ & $53.5 \pm 0.2$ & $4 \pm 11$ & $33 \pm 12$ & $33 \pm 12$ \\
\hline A1N & 110.4 & 1.8 & $66.7 \pm 0.2$ & $-108.1 \pm 0.2$ & $13 \pm 10$ & $5 \pm 13$ & $14 \pm 11$ \\
\hline A1N & 108.3 & 1.7 & $32.7 \pm 0.2$ & $26.1 \pm 0.5$ & & & \\
\hline A1N & 117.8 & 1.7 & $31.4 \pm 0.2$ & $20.2 \pm 0.2$ & & & \\
\hline $\mathrm{A} 1 \mathrm{~N}$ & 110.2 & 1.6 & $-23.8 \pm 0.2$ & $46.9 \pm 0.2$ & $7 \pm 10$ & $43 \pm 13$ & $44 \pm 12$ \\
\hline
\end{tabular}


L. Moscadelli et al.: VLBI of molecular masers towards G24.78+0.08, Online Material p 4

Table 5. continued.

\begin{tabular}{|c|c|c|c|c|c|c|c|}
\hline Cluster & $\begin{array}{r}V_{\text {LSR }} \\
\left(\mathrm{km} \mathrm{s}^{-1}\right)\end{array}$ & $\begin{array}{l}F_{\text {int }} \\
(\mathrm{Jy})\end{array}$ & $\begin{array}{c}\Delta \alpha \\
(\mathrm{mas})\end{array}$ & $\begin{array}{c}\Delta \delta \\
(\mathrm{mas})\end{array}$ & $\begin{array}{c}V_{x} \\
\left(\mathrm{~km} \mathrm{~s}^{-1}\right)\end{array}$ & $\begin{array}{c}V_{y} \\
\left(\mathrm{~km} \mathrm{~s}^{-1}\right)\end{array}$ & $\begin{array}{c}V_{\text {mod }} \\
\left(\mathrm{km} \mathrm{s}^{-1}\right)\end{array}$ \\
\hline A1N & 113.6 & 1.6 & $-29.9 \pm 0.3$ & $45.0 \pm 0.3$ & $-6 \pm 15$ & $38 \pm 13$ & $38 \pm 13$ \\
\hline $\mathrm{A} 1 \mathrm{~N}$ & 107.2 & 1.5 & $32.8 \pm 0.2$ & $27.8 \pm 0.2$ & & & \\
\hline A1N & 116.4 & 1.4 & $30.7 \pm 0.2$ & $32.1 \pm 0.4$ & $62 \pm 22$ & $30 \pm 35$ & $69 \pm 25$ \\
\hline A1N & 113.3 & 1.4 & $30.5 \pm 0.2$ & $33.4 \pm 0.3$ & & & \\
\hline A1N & 111.6 & 1.3 & $32.8 \pm 0.2$ & $25.6 \pm 0.3$ & & & \\
\hline A1N & 116.6 & 1.2 & $30.9 \pm 0.2$ & $32.9 \pm 0.3$ & & & \\
\hline A1N & 112.7 & 1.1 & $27.0 \pm 0.2$ & $6.9 \pm 0.3$ & & & \\
\hline $\mathrm{A} 1 \mathrm{~N}$ & 113.6 & 1.0 & $28.6 \pm 0.2$ & $35.4 \pm 0.3$ & $25 \pm 16$ & $62 \pm 20$ & $67 \pm 19$ \\
\hline A1N & 114.2 & 1.0 & $9.7 \pm 0.2$ & $44.4 \pm 0.2$ & & & \\
\hline A1N & 107.5 & 0.9 & $-3.9 \pm 0.2$ & $7.0 \pm 0.2$ & & & \\
\hline A1N & 108.4 & 0.9 & $6.1 \pm 0.2$ & $1.4 \pm 0.3$ & $9 \pm 11$ & $13 \pm 13$ & $17 \pm 12$ \\
\hline A1N & 113.7 & 0.6 & $31.4 \pm 0.3$ & $32.1 \pm 0.3$ & & & \\
\hline A1N & 117.6 & 0.6 & $29.9 \pm 0.2$ & $18.6 \pm 0.2$ & $29 \pm 21$ & $36 \pm 29$ & $46 \pm 26$ \\
\hline $\mathrm{A} 1 \mathrm{~N}$ & 108.6 & 0.5 & $33.4 \pm 0.2$ & $27.7 \pm 0.2$ & & & \\
\hline A1N & 114.7 & 0.4 & $-117.0 \pm 0.2$ & $55.2 \pm 0.2$ & $-23 \pm 21$ & $32 \pm 28$ & $40 \pm 26$ \\
\hline A1N & 105.6 & 0.4 & $-159.8 \pm 0.2$ & $88.2 \pm 0.3$ & & & \\
\hline A1N & 110.3 & 0.4 & $-22.7 \pm 0.2$ & $46.9 \pm 0.3$ & & & \\
\hline A1N & 114.8 & 0.4 & $19.8 \pm 0.2$ & $37.9 \pm 0.2$ & & & \\
\hline A1N & 109.4 & 0.4 & $30.4 \pm 0.2$ & $32.6 \pm 0.3$ & & & \\
\hline A1N & 116.2 & 0.3 & $30.4 \pm 0.2$ & $20.2 \pm 0.3$ & & & \\
\hline A1N & 108.7 & 0.3 & $36.6 \pm 0.2$ & $-100.0 \pm 0.2$ & $21 \pm 11$ & $18 \pm 13$ & $28 \pm 12$ \\
\hline A1N & 116.1 & 0.3 & $32.9 \pm 0.2$ & $27.0 \pm 0.3$ & & & \\
\hline A1N & 105.1 & 0.2 & $-23.8 \pm 0.2$ & $-79.5 \pm 0.3$ & $-27 \pm 21$ & $17 \pm 29$ & $32 \pm 24$ \\
\hline A1N & 110.3 & 0.2 & $63.6 \pm 0.2$ & $-82.2 \pm 0.3$ & $12 \pm 11$ & $24 \pm 13$ & $27 \pm 12$ \\
\hline A1N & 110.3 & 0.2 & $63.7 \pm 0.2$ & $-84.6 \pm 0.3$ & $-8 \pm 21$ & $35 \pm 28$ & $36 \pm 28$ \\
\hline A1N & 114.5 & 0.2 & $27.7 \pm 0.2$ & $11.3 \pm 0.3$ & & & \\
\hline A1N & 108.6 & 0.2 & $5.4 \pm 0.2$ & $1.1 \pm 0.3$ & & & \\
\hline $\mathrm{A} 1 \mathrm{~N}$ & 115.4 & 0.2 & $18.7 \pm 0.2$ & $-72.3 \pm 0.3$ & & & \\
\hline A1N & 109.7 & 0.2 & $45.9 \pm 0.2$ & $-141.1 \pm 0.2$ & & & \\
\hline A1N & 109.3 & 0.2 & $-31.4 \pm 0.2$ & $45.7 \pm 0.3$ & $14 \pm 10$ & $52 \pm 13$ & $54 \pm 12$ \\
\hline A1N & 107.3 & 0.1 & $31.7 \pm 0.2$ & $30.5 \pm 0.3$ & & & \\
\hline A1N & 115.5 & 0.1 & $22.3 \pm 0.2$ & $5.6 \pm 0.3$ & & & \\
\hline A1N & 117.6 & 0.1 & $30.0 \pm 0.2$ & $33.3 \pm 0.3$ & & & \\
\hline A1N & 107.5 & 0.1 & $39.4 \pm 0.2$ & $22.1 \pm 0.3$ & & & \\
\hline A1N & 107.7 & 0.1 & $-24.2 \pm 0.2$ & $44.6 \pm 0.3$ & & & \\
\hline A1N & 106.2 & 0.08 & $30.5 \pm 0.2$ & $32.5 \pm 0.3$ & & & \\
\hline A1N & 105.0 & 0.07 & $-5.5 \pm 0.2$ & $7.3 \pm 0.3$ & & & \\
\hline A1N & 116.7 & 0.05 & $27.4 \pm 0.2$ & $15.1 \pm 0.3$ & & & \\
\hline A1N & 91.6 & 0.05 & $120.2 \pm 0.3$ & $135.0 \pm 0.4$ & & & \\
\hline A1S & 107.5 & 11.3 & $-369.4 \pm 0.2$ & $-521.3 \pm 0.2$ & & & \\
\hline A1S & 107.7 & 2.1 & $-368.2 \pm 0.2$ & $-521.4 \pm 0.2$ & & & \\
\hline A1S & 106.1 & 0.8 & $-368.4 \pm 0.2$ & $-521.2 \pm 0.2$ & & & \\
\hline A1S & 109.8 & 0.6 & $-397.4 \pm 0.2$ & $-519.8 \pm 0.3$ & & & \\
\hline A1S & 109.4 & 0.4 & $-397.2 \pm 0.2$ & $-521.0 \pm 0.2$ & & & \\
\hline A1S & 109.9 & 0.3 & $-397.8 \pm 0.2$ & $-517.8 \pm 0.2$ & & & \\
\hline A1S & 109.2 & 0.2 & $-395.9 \pm 0.2$ & $-517.4 \pm 0.2$ & & & \\
\hline A1S & 108.5 & 0.1 & $-396.2 \pm 0.2$ & $-524.2 \pm 0.3$ & & & \\
\hline A2 & 105.5 & 5.0 & $-1165.7 \pm 0.2$ & $647.2 \pm 0.2$ & $17 \pm 11$ & $5 \pm 12$ & $18 \pm 11$ \\
\hline $\mathrm{A} 2$ & 106.7 & 0.9 & $-1475.1 \pm 0.2$ & $655.5 \pm 0.3$ & $-10 \pm 11$ & $12 \pm 14$ & $16 \pm 12$ \\
\hline $\mathrm{A} 2$ & 106.4 & 0.8 & $-1474.8 \pm 0.2$ & $656.6 \pm 0.3$ & & & \\
\hline $\mathrm{A} 2$ & 106.8 & 0.7 & $-1325.0 \pm 0.2$ & $636.9 \pm 0.3$ & & & \\
\hline A2 & 105.5 & 0.5 & $-1330.3 \pm 0.2$ & $648.4 \pm 0.3$ & $-3 \pm 10$ & $25 \pm 13$ & $25 \pm 13$ \\
\hline A2 & 105.7 & 0.5 & $-1474.8 \pm 0.2$ & $657.5 \pm 0.3$ & $-3 \pm 10$ & $5 \pm 14$ & $6 \pm 13$ \\
\hline A2 & 105.9 & 0.1 & $-1171.5 \pm 0.2$ & $647.4 \pm 0.3$ & $-6 \pm 16$ & $7 \pm 15$ & $9 \pm 15$ \\
\hline
\end{tabular}


Table 5. continued.

\begin{tabular}{|c|c|c|c|c|c|c|c|}
\hline Cluster & $\begin{array}{r}V_{\text {LSR }} \\
\left(\mathrm{km} \mathrm{s}^{-1}\right)\end{array}$ & $\begin{array}{l}F_{\text {int }} \\
(\mathrm{Jy})\end{array}$ & $\begin{array}{c}\Delta \alpha \\
\text { (mas) }\end{array}$ & $\begin{array}{c}\Delta \delta \\
(\mathrm{mas})\end{array}$ & $\begin{array}{c}V_{x} \\
\left(\mathrm{~km} \mathrm{~s}^{-1}\right)\end{array}$ & $\begin{array}{c}V_{y} \\
\left(\mathrm{~km} \mathrm{~s}^{-1}\right)\end{array}$ & $\begin{array}{c}V_{\text {mod }} \\
\left(\mathrm{km} \mathrm{s}^{-1}\right)\end{array}$ \\
\hline $\mathrm{A} / \mathrm{B}$ & 94.4 & 0.5 & $720.2 \pm 0.2$ & $-1182.1 \pm 0.3$ & $10 \pm 11$ & $57 \pm 13$ & $58 \pm 13$ \\
\hline $\mathrm{A} / \mathrm{B}$ & 90.3 & 0.5 & $721.6 \pm 0.2$ & $-1182.8 \pm 0.2$ & $-10 \pm 21$ & $79 \pm 28$ & $79 \pm 28$ \\
\hline $\mathrm{A} / \mathrm{B}$ & 93.5 & 0.4 & $721.2 \pm 0.2$ & $-1182.0 \pm 0.3$ & $36 \pm 11$ & $59 \pm 13$ & $70 \pm 12$ \\
\hline $\mathrm{A} / \mathrm{B}$ & 90.2 & 0.3 & $721.1 \pm 0.2$ & $-1181.2 \pm 0.2$ & & & \\
\hline $\mathrm{A} / \mathrm{B}$ & 89.9 & 0.3 & $720.5 \pm 0.2$ & $-1182.9 \pm 0.2$ & & & \\
\hline $\mathrm{A} / \mathrm{B}$ & 95.4 & 0.2 & $712.0 \pm 0.2$ & $-1195.8 \pm 0.3$ & & & \\
\hline $\mathrm{A} / \mathrm{B}$ & 92.6 & 0.1 & $713.0 \pm 0.2$ & $-1196.6 \pm 0.3$ & & & \\
\hline $\mathrm{A} / \mathrm{B}$ & 90.6 & 0.1 & $713.1 \pm 0.2$ & $-1194.0 \pm 0.3$ & $-4 \pm 10$ & $36 \pm 13$ & $36 \pm 13$ \\
\hline $\mathrm{A} / \mathrm{B}$ & 89.8 & 0.09 & $712.9 \pm 0.2$ & $-1195.3 \pm 0.2$ & & & \\
\hline B & 105.5 & 0.3 & $1090.5 \pm 0.2$ & $-2778.3 \pm 0.3$ & & & \\
\hline B & 105.5 & 0.3 & $774.0 \pm 0.2$ & $-2757.5 \pm 0.3$ & & & \\
\hline B & 101.5 & 0.2 & $1107.0 \pm 0.2$ & $-2882.8 \pm 0.3$ & & & \\
\hline B & 104.7 & 0.1 & $1107.7 \pm 0.2$ & $-2882.7 \pm 0.3$ & $-90 \pm 22$ & $60 \pm 30$ & $109 \pm 25$ \\
\hline B & 91.7 & 0.1 & $1264.1 \pm 0.2$ & $-2738.6 \pm 0.3$ & $33 \pm 10$ & $145 \pm 13$ & $148 \pm 13$ \\
\hline B & 92.7 & 0.06 & $1361.2 \pm 0.3$ & $-2865.8 \pm 0.2$ & & & \\
\hline $\mathrm{E}$ & 93.9 & 0.5 & $-2388.2 \pm 0.2$ & $-684.6 \pm 0.2$ & $-15 \pm 21$ & $24 \pm 28$ & $28 \pm 27$ \\
\hline $\mathrm{E}$ & 102.2 & 0.4 & $-2389.9 \pm 0.2$ & $-679.7 \pm 0.2$ & & & \\
\hline $\mathrm{E}$ & 104.9 & 0.2 & $-2390.2 \pm 0.2$ & $-680.4 \pm 0.3$ & & & \\
\hline $\mathrm{E}$ & 93.9 & 0.2 & $-2387.9 \pm 0.2$ & $-684.9 \pm 0.3$ & & & \\
\hline $\mathrm{E}$ & 93.7 & 0.2 & $-2388.0 \pm 0.2$ & $-685.4 \pm 0.2$ & & & \\
\hline $\mathrm{E}$ & 103.3 & 0.2 & $-2381.6 \pm 0.2$ & $-671.6 \pm 0.2$ & & & \\
\hline $\mathrm{E}$ & 102.9 & 0.2 & $-2389.9 \pm 0.2$ & $-679.9 \pm 0.3$ & $7 \pm 22$ & $54 \pm 29$ & $55 \pm 28$ \\
\hline $\mathrm{E}$ & 95.8 & 0.2 & $-2388.5 \pm 0.2$ & $-684.6 \pm 0.3$ & & & \\
\hline $\mathrm{E}$ & 103.0 & 0.2 & $-2389.5 \pm 0.2$ & $-677.4 \pm 0.3$ & & & \\
\hline $\mathrm{E}$ & 104.7 & 0.1 & $-2390.6 \pm 0.2$ & $-680.3 \pm 0.3$ & & & \\
\hline $\mathrm{E}$ & 97.7 & 0.1 & $-2388.7 \pm 0.2$ & $-684.9 \pm 0.2$ & & & \\
\hline
\end{tabular}


Table 6. Parameters of VLBA water maser features in core C. For each identified feature, Col. 1 indicates the harboring maser subcluster; Cols. 2 and 3 the LSR velocity and the integrated flux density of the highest-intensity channel; Cols. 4 and 5 the positional (RA and Dec) offsets (with the associated errors), evaluated with respect to the derived absolute position of the reference feature $\left(\alpha(J 2000)=18^{\mathrm{h}} 36^{\mathrm{m}} 13 \mathrm{3}^{\mathrm{s}} .1140\right.$, $\left.\delta(J 2000)=-7^{\circ} 12^{\prime} 7^{\prime \prime} \cdot 528\right)$; Cols. 6-8 the projected components along the RA and Dec axes and the absolute value of the derived proper motions (together with the associated errors).

\begin{tabular}{|c|c|c|c|c|c|c|c|}
\hline Cluster & $\begin{array}{c}V_{\mathrm{LSR}} \\
\left(\mathrm{km} \mathrm{s}^{-1}\right)\end{array}$ & $\begin{array}{l}F_{\text {int }} \\
(\mathrm{Jy})\end{array}$ & $\begin{array}{l}\Delta \alpha \\
(\mathrm{mas})\end{array}$ & $\begin{array}{c}\Delta \delta \\
(\mathrm{mas})\end{array}$ & $\begin{array}{c}V_{x} \\
\left(\mathrm{~km} \mathrm{~s}^{-1}\right)\end{array}$ & $\begin{array}{c}V_{y} \\
\left(\mathrm{~km} \mathrm{~s}^{-1}\right)\end{array}$ & $\begin{array}{c}V_{\text {mod }} \\
\left(\mathrm{km} \mathrm{s}^{-1}\right)\end{array}$ \\
\hline $\mathrm{C} 1$ & 114.5 & 4.8 & $0.0 \pm 0.2$ & $0.0 \pm 0.2$ & $-80 \pm 9$ & $-101 \pm 9$ & $129 \pm 9$ \\
\hline $\mathrm{C} 1$ & 114.7 & 1.5 & $162.4 \pm 0.2$ & $241.3 \pm 0.2$ & $-55 \pm 10$ & $-153 \pm 10$ & $163 \pm 10$ \\
\hline $\mathrm{C} 1$ & 110.8 & 1.3 & $81.2 \pm 0.2$ & $87.0 \pm 0.2$ & & & \\
\hline $\mathrm{C} 1$ & 107.7 & 1.1 & $41.2 \pm 0.2$ & $33.5 \pm 0.2$ & & & \\
\hline $\mathrm{C} 1$ & 115.1 & 0.8 & $163.3 \pm 0.2$ & $242.6 \pm 0.2$ & $-18 \pm 18$ & $-76 \pm 19$ & $78 \pm 19$ \\
\hline $\mathrm{C} 1$ & 116.7 & 0.8 & $18.6 \pm 0.2$ & $-8.4 \pm 0.2$ & $-49 \pm 18$ & $-68 \pm 19$ & $84 \pm 18$ \\
\hline $\mathrm{C} 1$ & 114.2 & 0.3 & $165.9 \pm 0.2$ & $254.2 \pm 0.2$ & & & \\
\hline $\mathrm{C} 1$ & 114.1 & 0.3 & $166.0 \pm 0.2$ & $249.1 \pm 0.2$ & & & \\
\hline $\mathrm{C} 1$ & 116.5 & 0.2 & $157.6 \pm 0.2$ & $238.3 \pm 0.2$ & $-51 \pm 18$ & $-93 \pm 19$ & $107 \pm 19$ \\
\hline $\mathrm{C} 1$ & 117.9 & 0.2 & $55.1 \pm 0.2$ & $-10.7 \pm 0.2$ & & & \\
\hline $\mathrm{C} 1$ & 107.6 & 0.1 & $58.7 \pm 0.2$ & $-22.5 \pm 0.2$ & & & \\
\hline $\mathrm{C} 1$ & 121.5 & 0.1 & $150.0 \pm 0.2$ & $257.0 \pm 0.2$ & & & \\
\hline $\mathrm{C} 1$ & 123.8 & 0.07 & $150.4 \pm 0.2$ & $258.1 \pm 0.2$ & & & \\
\hline $\mathrm{C} 1$ & 127.1 & 0.06 & $150.0 \pm 0.2$ & $290.2 \pm 0.2$ & & & \\
\hline $\mathrm{C} 1$ & 107.2 & 0.06 & $40.6 \pm 0.2$ & $37.9 \pm 0.2$ & & & \\
\hline $\mathrm{C} 2$ & 102.3 & 1.6 & $20.5 \pm 0.2$ & $372.7 \pm 0.2$ & & & \\
\hline $\mathrm{C} 2$ & 105.0 & 1.6 & $18.4 \pm 0.2$ & $370.5 \pm 0.2$ & & & \\
\hline $\mathrm{C} 2$ & 124.6 & 1.0 & $59.6 \pm 0.2$ & $361.6 \pm 0.2$ & $-24 \pm 14$ & $-178 \pm 14$ & $180 \pm 14$ \\
\hline $\mathrm{C} 2$ & 119.3 & 0.9 & $52.0 \pm 0.2$ & $365.2 \pm 0.2$ & $-15 \pm 11$ & $-139 \pm 10$ & $140 \pm 10$ \\
\hline $\mathrm{C} 2$ & 124.2 & 0.9 & $63.5 \pm 0.2$ & $357.3 \pm 0.3$ & & & \\
\hline $\mathrm{C} 2$ & 107.3 & 0.9 & $18.4 \pm 0.2$ & $372.5 \pm 0.2$ & & & \\
\hline $\mathrm{C} 2$ & 109.2 & 0.8 & $17.7 \pm 0.2$ & $370.5 \pm 0.2$ & & & \\
\hline $\mathrm{C} 2$ & 125.5 & 0.7 & $59.2 \pm 0.2$ & $361.8 \pm 0.2$ & & & \\
\hline $\mathrm{C} 2$ & 125.3 & 0.7 & $63.5 \pm 0.2$ & $357.4 \pm 0.2$ & $0 \pm 14$ & $-199 \pm 14$ & $199 \pm 14$ \\
\hline $\mathrm{C} 2$ & 101.1 & 0.5 & $16.1 \pm 0.2$ & $372.7 \pm 0.2$ & & & \\
\hline $\mathrm{C} 2$ & 103.4 & 0.5 & $29.5 \pm 0.2$ & $364.6 \pm 0.2$ & $25 \pm 19$ & $-94 \pm 20$ & $98 \pm 20$ \\
\hline $\mathrm{C} 2$ & 120.6 & 0.4 & $62.6 \pm 0.2$ & $359.6 \pm 0.2$ & & & \\
\hline $\mathrm{C} 2$ & 94.9 & 0.4 & $20.0 \pm 0.2$ & $372.7 \pm 0.2$ & & & \\
\hline $\mathrm{C} 2$ & 98.4 & 0.4 & $19.5 \pm 0.2$ & $373.0 \pm 0.2$ & & & \\
\hline $\mathrm{C} 2$ & 96.3 & 0.4 & $18.1 \pm 0.2$ & $373.4 \pm 0.2$ & $-127 \pm 10$ & $-84 \pm 12$ & $152 \pm 11$ \\
\hline $\mathrm{C} 2$ & 124.0 & 0.3 & $60.4 \pm 0.2$ & $361.0 \pm 0.2$ & $-1 \pm 14$ & $-190 \pm 15$ & $190 \pm 15$ \\
\hline $\mathrm{C} 2$ & 90.8 & 0.3 & $29.7 \pm 0.2$ & $366.0 \pm 0.2$ & & & \\
\hline $\mathrm{C} 2$ & 103.2 & 0.3 & $19.1 \pm 0.2$ & $372.4 \pm 0.2$ & $-97 \pm 18$ & $26 \pm 19$ & $101 \pm 18$ \\
\hline $\mathrm{C} 2$ & 97.5 & 0.3 & $19.7 \pm 0.2$ & $370.1 \pm 0.2$ & & & \\
\hline $\mathrm{C} 2$ & 121.7 & 0.3 & $63.2 \pm 0.2$ & $358.9 \pm 0.2$ & & & \\
\hline $\mathrm{C} 2$ & 101.6 & 0.3 & $16.8 \pm 0.2$ & $372.5 \pm 0.2$ & & & \\
\hline $\mathrm{C} 2$ & 108.0 & 0.2 & $20.3 \pm 0.2$ & $372.6 \pm 0.2$ & & & \\
\hline $\mathrm{C} 2$ & 106.5 & 0.2 & $18.0 \pm 0.2$ & $367.3 \pm 0.2$ & & & \\
\hline $\mathrm{C} 2$ & 108.7 & 0.2 & $16.3 \pm 0.2$ & $371.1 \pm 0.2$ & & & \\
\hline $\mathrm{C} 2$ & 106.0 & 0.2 & $18.2 \pm 0.2$ & $374.0 \pm 0.2$ & & & \\
\hline $\mathrm{C} 2$ & 100.5 & 0.2 & $18.0 \pm 0.2$ & $372.4 \pm 0.2$ & & & \\
\hline $\mathrm{C} 2$ & 124.9 & 0.2 & $62.5 \pm 0.2$ & $356.8 \pm 0.2$ & & & \\
\hline $\mathrm{C} 2$ & 97.8 & 0.1 & $15.0 \pm 0.2$ & $368.9 \pm 0.2$ & & & \\
\hline $\mathrm{C} 2$ & 106.8 & 0.1 & $19.7 \pm 0.2$ & $372.7 \pm 0.2$ & & & \\
\hline $\mathrm{C} 2$ & 125.7 & 0.1 & $62.7 \pm 0.2$ & $358.8 \pm 0.2$ & & & \\
\hline $\mathrm{C} 2$ & 97.1 & 0.1 & $3.3 \pm 0.2$ & $385.1 \pm 0.2$ & & & \\
\hline $\mathrm{C} 2$ & 126.5 & 0.1 & $63.8 \pm 0.2$ & $355.5 \pm 0.2$ & & & \\
\hline $\mathrm{C} 2$ & 91.4 & 0.09 & $17.5 \pm 0.2$ & $372.5 \pm 0.3$ & & & \\
\hline $\mathrm{C} 2$ & 95.1 & 0.08 & $-1.3 \pm 0.2$ & $382.9 \pm 0.2$ & & & \\
\hline $\mathrm{C} 2$ & 119.6 & 0.07 & $61.3 \pm 0.2$ & $361.2 \pm 0.2$ & & & \\
\hline $\mathrm{C} 2$ & 107.9 & 0.05 & $17.4 \pm 0.2$ & $373.1 \pm 0.2$ & & & \\
\hline $\mathrm{C} 3$ & 54.0 & 59.9 & $-161.0 \pm 0.2$ & $364.7 \pm 0.2$ & $-39 \pm 20$ & $-74 \pm 20$ & $84 \pm 20$ \\
\hline $\mathrm{C} 3$ & 60.8 & 16.4 & $-160.0 \pm 0.2$ & $366.9 \pm 0.2$ & $-58 \pm 10$ & $-123 \pm 11$ & $136 \pm 11$ \\
\hline $\mathrm{C} 3$ & 48.9 & 11.8 & $-161.8 \pm 0.2$ & $364.0 \pm 0.2$ & & & \\
\hline $\mathrm{C} 3$ & 56.3 & 7.2 & $-160.7 \pm 0.2$ & $366.8 \pm 0.2$ & $-61 \pm 15$ & $-193 \pm 16$ & $202 \pm 16$ \\
\hline $\mathrm{C} 3$ & 57.2 & 6.0 & $-161.0 \pm 0.2$ & $365.2 \pm 0.2$ & & & \\
\hline $\mathrm{C} 3$ & 73.0 & 4.0 & $-163.0 \pm 0.2$ & $380.2 \pm 0.2$ & & & \\
\hline C3 & 43.8 & 3.1 & $-161.4 \pm 0.2$ & $365.3 \pm 0.2$ & & & \\
\hline $\mathrm{C} 3$ & 96.9 & 2.4 & $-215.6 \pm 0.2$ & $410.3 \pm 0.2$ & $-68 \pm 10$ & $-148 \pm 10$ & $163 \pm 10$ \\
\hline
\end{tabular}


Table 6. continued.

\begin{tabular}{|c|c|c|c|c|c|c|c|}
\hline Cluster & $\begin{array}{c}V_{\mathrm{LSR}} \\
\left(\mathrm{km} \mathrm{s}^{-1}\right)\end{array}$ & $\begin{array}{l}F_{\text {int }} \\
(\mathrm{Jy})\end{array}$ & $\begin{array}{l}\Delta \alpha \\
(\mathrm{mas})\end{array}$ & $\begin{array}{c}\Delta \delta \\
(\mathrm{mas})\end{array}$ & $\begin{array}{c}V_{x} \\
\left(\mathrm{~km} \mathrm{~s}^{-1}\right)\end{array}$ & $\begin{array}{c}V_{y} \\
\left(\mathrm{~km} \mathrm{~s}^{-1}\right)\end{array}$ & $\begin{array}{c}V_{\text {mod }} \\
\left(\mathrm{km} \mathrm{s}^{-1}\right)\end{array}$ \\
\hline C3 & 43.0 & 2.4 & $-160.5 \pm 0.2$ & $365.9 \pm 0.2$ & & & \\
\hline $\mathrm{C} 3$ & 57.1 & 2.3 & $-161.5 \pm 0.2$ & $364.1 \pm 0.2$ & & & \\
\hline $\mathrm{C} 3$ & 97.0 & 1.6 & $-215.1 \pm 0.2$ & $405.9 \pm 0.2$ & & & \\
\hline $\mathrm{C} 3$ & 83.1 & 1.4 & $-212.6 \pm 0.2$ & $409.5 \pm 0.2$ & & & \\
\hline $\mathrm{C} 3$ & 48.8 & 1.2 & $-161.8 \pm 0.2$ & $364.8 \pm 0.2$ & $-67 \pm 14$ & $-177 \pm 14$ & $190 \pm 14$ \\
\hline $\mathrm{C} 3$ & 44.0 & 1.0 & $-160.1 \pm 0.2$ & $366.3 \pm 0.2$ & & & \\
\hline C3 & 47.9 & 0.9 & $-162.3 \pm 0.2$ & $366.5 \pm 0.2$ & $-53 \pm 14$ & $-151 \pm 15$ & $160 \pm 15$ \\
\hline C3 & 94.8 & 0.9 & $-216.5 \pm 0.2$ & $406.7 \pm 0.2$ & & & \\
\hline C3 & 39.5 & 0.8 & $-161.4 \pm 0.2$ & $365.6 \pm 0.2$ & & & \\
\hline C3 & 96.0 & 0.8 & $-216.9 \pm 0.2$ & $407.1 \pm 0.2$ & & & \\
\hline C3 & 109.6 & 0.8 & $-161.4 \pm 0.2$ & $371.7 \pm 0.2$ & & & \\
\hline $\mathrm{C} 3$ & 109.9 & 0.8 & $-536.0 \pm 0.2$ & $629.0 \pm 0.2$ & & & \\
\hline C3 & 88.1 & 0.7 & $-161.0 \pm 0.2$ & $374.1 \pm 0.2$ & & & \\
\hline C3 & 37.8 & 0.7 & $-160.7 \pm 0.2$ & $366.8 \pm 0.2$ & & & \\
\hline C3 & 58.2 & 0.6 & $-162.8 \pm 0.2$ & $361.7 \pm 0.2$ & & & \\
\hline $\mathrm{C} 3$ & 56.1 & 0.6 & $-158.8 \pm 0.2$ & $368.6 \pm 0.2$ & $-52 \pm 19$ & $-114 \pm 23$ & $126 \pm 22$ \\
\hline $\mathrm{C} 3$ & 100.1 & 0.5 & $-216.8 \pm 0.2$ & $410.9 \pm 0.2$ & $-67 \pm 14$ & $-181 \pm 17$ & $193 \pm 17$ \\
\hline $\mathrm{C} 3$ & 71.0 & 0.5 & $-215.6 \pm 0.2$ & $412.4 \pm 0.2$ & $-62 \pm 14$ & $-162 \pm 14$ & $174 \pm 14$ \\
\hline C3 & 116.6 & 0.5 & $-160.1 \pm 0.2$ & $365.3 \pm 0.2$ & & & \\
\hline $\mathrm{C} 3$ & 105.2 & 0.5 & $-159.2 \pm 0.2$ & $367.1 \pm 0.2$ & & & \\
\hline $\mathrm{C} 3$ & 58.0 & 0.4 & $-160.4 \pm 0.2$ & $365.1 \pm 0.2$ & & & \\
\hline C3 & 99.6 & 0.3 & $-159.8 \pm 0.2$ & $366.1 \pm 0.2$ & & & \\
\hline $\mathrm{C} 3$ & 34.6 & 0.3 & $-161.0 \pm 0.2$ & $368.0 \pm 0.2$ & & & \\
\hline C3 & 98.1 & 0.3 & $-211.9 \pm 0.2$ & $410.9 \pm 0.2$ & $-26 \pm 18$ & $-96 \pm 19$ & $99 \pm 19$ \\
\hline C3 & 64.4 & 0.3 & $-160.5 \pm 0.2$ & $366.5 \pm 0.2$ & & & \\
\hline C3 & 95.4 & 0.3 & $-214.0 \pm 0.2$ & $409.0 \pm 0.2$ & $-52 \pm 14$ & $-199 \pm 14$ & $205 \pm 14$ \\
\hline C3 & 76.7 & 0.3 & $-176.3 \pm 0.2$ & $390.4 \pm 0.2$ & & & \\
\hline C3 & 42.1 & 0.3 & $-163.0 \pm 0.2$ & $365.8 \pm 0.2$ & & & \\
\hline C3 & 52.1 & 0.3 & $-162.5 \pm 0.2$ & $362.1 \pm 0.2$ & & & \\
\hline C3 & 70.3 & 0.2 & $-162.4 \pm 0.2$ & $359.9 \pm 0.2$ & & & \\
\hline C3 & 49.9 & 0.2 & $-164.8 \pm 0.2$ & $366.0 \pm 0.2$ & & & \\
\hline C3 & 80.4 & 0.2 & $-214.9 \pm 0.2$ & $409.6 \pm 0.2$ & & & \\
\hline C3 & 96.9 & 0.2 & $-216.8 \pm 0.2$ & $411.4 \pm 0.2$ & & & \\
\hline C3 & 42.0 & 0.2 & $-162.6 \pm 0.2$ & $365.3 \pm 0.2$ & & & \\
\hline C3 & 74.0 & 0.2 & $-175.8 \pm 0.2$ & $390.1 \pm 0.2$ & & & \\
\hline C3 & 68.3 & 0.2 & $-162.8 \pm 0.2$ & $379.9 \pm 0.2$ & & & \\
\hline $\mathrm{C} 3$ & 87.7 & 0.2 & $-182.0 \pm 0.2$ & $392.3 \pm 0.2$ & & & \\
\hline $\mathrm{C} 3$ & 68.9 & 0.2 & $-161.4 \pm 0.2$ & $364.2 \pm 0.2$ & & & \\
\hline $\mathrm{C} 3$ & 54.8 & 0.2 & $-162.5 \pm 0.2$ & $361.7 \pm 0.2$ & & & \\
\hline $\mathrm{C} 3$ & 82.7 & 0.2 & $-215.9 \pm 0.2$ & $412.6 \pm 0.2$ & & & \\
\hline C3 & 45.3 & 0.2 & $-162.5 \pm 0.2$ & $365.5 \pm 0.2$ & & & \\
\hline C3 & 105.6 & 0.2 & $-160.3 \pm 0.2$ & $365.6 \pm 0.2$ & & & \\
\hline C3 & 39.5 & 0.1 & $-161.4 \pm 0.2$ & $366.7 \pm 0.2$ & & & \\
\hline C3 & 97.7 & 0.1 & $-215.8 \pm 0.2$ & $406.0 \pm 0.2$ & & & \\
\hline $\mathrm{C} 3$ & 84.5 & 0.1 & $-182.5 \pm 0.2$ & $410.0 \pm 0.2$ & & & \\
\hline $\mathrm{C} 3$ & 43.4 & 0.1 & $-163.3 \pm 0.2$ & $363.5 \pm 0.2$ & & & \\
\hline C3 & 52.6 & 0.1 & $-165.3 \pm 0.2$ & $366.9 \pm 0.2$ & & & \\
\hline C3 & 83.5 & 0.1 & $-214.7 \pm 0.2$ & $411.0 \pm 0.2$ & & & \\
\hline $\mathrm{C} 3$ & 78.5 & 0.1 & $-163.3 \pm 0.2$ & $380.6 \pm 0.2$ & & & \\
\hline $\mathrm{C} 3$ & 48.0 & 0.1 & $-162.0 \pm 0.2$ & $364.3 \pm 0.2$ & & & \\
\hline C3 & 50.6 & 0.1 & $-160.9 \pm 0.2$ & $365.9 \pm 0.2$ & & & \\
\hline $\mathrm{C} 3$ & 38.0 & 0.08 & $-161.3 \pm 0.2$ & $365.9 \pm 0.2$ & & & \\
\hline C3 & 105.5 & 0.08 & $-161.2 \pm 0.2$ & $306.5 \pm 0.2$ & & & \\
\hline C3 & 82.2 & 0.07 & $-216.7 \pm 0.2$ & $428.4 \pm 0.2$ & & & \\
\hline $\mathrm{C} 3$ & 90.1 & 0.06 & $-212.3 \pm 0.2$ & $412.8 \pm 0.2$ & & & \\
\hline $\mathrm{C} 3$ & 53.8 & 0.05 & $-169.6 \pm 0.2$ & $399.3 \pm 0.2$ & & & \\
\hline $\mathrm{C} 3$ & 62.4 & 0.05 & $-163.6 \pm 0.2$ & $376.3 \pm 0.2$ & & & \\
\hline C3 & 85.2 & 0.04 & $-421.3 \pm 0.2$ & $715.4 \pm 0.3$ & & & \\
\hline
\end{tabular}




\section{Appendix A: Pressure and momentum-driven Shell model}

In this appendix we demonstrate that wind-driven expansion of an HII region may reproduce the observed HII radius and expansion velocity, provided the HII region age, $t_{0}$, is less than $\approx 80 \mathrm{yr}$. Details on the performed calculations and, in particular, on the derivation of $t_{0}$ will be given in Appendix B.

The interaction of the stellar wind with the surrounding medium is characterized by various evolutionary phases. Shortly after an initial phase of free expansion at the wind velocity, a high pressure bubble of shocked wind material expands against the surrounding ambient gas. The physical conditions of the thin, dense shell of swept-up interstellar matter are suitable for the excitation of water masers. In a later phase, the shocked wind bubble cools by thermal bremsstrahlung and its volume reduces till it becomes a thin shell pushed forward by the impinging wind. In the following discussion, we describe the evolution of a wind-driven bubble making use of the results derived by Castor et al. (1975) and Shull (1980). The wind is described in terms of mass loss rate $\dot{M}_{\mathrm{w}}=\left(10^{-6} M_{\odot} \mathrm{yr}^{-1}\right) \dot{M}_{-6}$ and velocity $V_{\mathrm{w}}=$ $\left(2000 \mathrm{~km} \mathrm{~s}^{-1}\right) V_{2000}$, impinging on a molecular cloud with $\mathrm{H}$ particle density $n_{\mathrm{H}}=\left(10^{5} \mathrm{~cm}^{-3}\right) n_{5}$. The wind mechanical luminosity is $L_{\mathrm{w}}=\left(10^{36} \mathrm{erg} \mathrm{s}^{-1}\right) L_{36}$, where $L_{36}=(1.27) \dot{M}_{-6} V_{2000}^{2}$. Following Lamers \& Leitherer (1993), plausible ranges of variation for the wind parameters for a B0-08 star, are $1 \leq \dot{M}_{-6} \leq 5$, $0.75 \leq V_{2000} \leq 1.25$, and $1 \leq L_{36} \leq 10$. As a plausible range of variation for the $\mathrm{H}$ number density of a massive molecular core we take $1 \leq n_{5} \leq 1000$ (see e.g. Kurtz et al. 2000).

The cooling time of the shocked wind material is $t_{\mathrm{c}}=$ $2.28 t_{\mathrm{cr}}$, with $t_{\mathrm{cr}}$ given by:

$t_{\text {cr }}=\left(10^{4} \mathrm{yr}\right) L_{36}^{-1 / 8} n_{5}^{-9 / 8} K_{0}^{-15 / 16}$

where $K_{0} \approx 1$ is the coefficient of the relation giving the dependence of the thermal conductivity on the gas temperature, $\kappa(T)=$ $\left(6 \times 10^{-7}\right) T^{5 / 2} K_{0} \operatorname{erg~cm}^{-1} \mathrm{~s}^{-1} \mathrm{~K}^{-1}$ (Shull 1980).

Rewriting the Eqs. (11) and (12) of Shull (1980), at time $t=t_{\mathrm{cr}}$, the radius and the velocity of the expanding shell are:

$$
\begin{aligned}
& R_{\text {cr }}=(34034 \mathrm{AU}) L_{36}^{1 / 8} n_{5}^{-7 / 8} K_{0}^{-9 / 16} \\
& V_{\text {cr }}=\left(9.64 \mathrm{~km} \mathrm{~s}^{-1}\right) L_{36}^{1 / 4} n_{5}^{1 / 4} K_{0}^{3 / 8} .
\end{aligned}
$$

For $t \leq t_{\mathrm{cr}}$, when the high pressure inside the shocked wind bubble drives the expansion, the shell radius and velocity vary with time as:

$$
\begin{aligned}
& R_{\mathrm{s}}=R_{\mathrm{cr}}\left(\frac{t}{t_{\mathrm{cr}}}\right)^{3 / 5} \\
& V_{\mathrm{s}}=V_{\mathrm{cr}}\left(\frac{t}{t_{\mathrm{cr}}}\right)^{-2 / 5} .
\end{aligned}
$$

For $t \geq t_{\mathrm{cr}}$, the momentum of the wind drives the shell. Making use of Eq. (17) of Shull (1980), the radius and the velocity of the shell can be expressed as:

$$
\begin{aligned}
& R_{\mathrm{s}}=R_{\mathrm{cr}}\left[1+\frac{12}{5}\left(\frac{t}{t_{\mathrm{cr}}}-1\right)+C_{1}\left(\frac{t}{t_{\mathrm{cr}}}-1\right)^{2}\right]^{1 / 4} \\
& V_{\mathrm{s}}=V_{\mathrm{cr}} \frac{\frac{5}{6} C_{1}\left(\frac{t}{t_{\mathrm{cr}}}-1\right)+1}{\left(\frac{R_{\mathrm{s}}}{R_{\mathrm{cr}}}\right)^{3}}
\end{aligned}
$$

where the coefficient $C_{1}$ is given by the equation:

$$
\begin{aligned}
C_{1} & =2.75 \times 10^{-2} \dot{M}_{-6} V_{2000}^{-2} n_{5}^{1 / 4} K_{0}^{3 / 8} \\
& =2.17 \times 10^{-2} L_{36} V_{2000}^{-4} n_{5}^{1 / 4} K_{0}^{3 / 8} .
\end{aligned}
$$

One can note that both $t_{\mathrm{cr}}$ and $R_{\mathrm{cr}}$ depend mostly on the ambient density, $n_{5}$, and very little on the wind mechanical luminosity, $L_{36}$.

In Appendix B.1, we show that the pressure-driven expansion can reproduce the observed values of shell radius, $R_{\mathrm{s}}=R_{0}$, and velocity, $V_{\mathrm{s}}=V_{0}$, only if the ambient density and wind luminosity satisfy Eq. (B.2). Using $R_{0}=500 \mathrm{AU}$ and $V_{0}=40 \mathrm{~km} \mathrm{~s}^{-1}$, for $L_{36}=1$ one derives $n_{5}=65$. The time $t_{0}$ at which the radius and the velocity of the pressure-driven bubble match the observed values $R_{0}$ and $V_{0}$ is given by Eq. (B.7). Using $R_{0}=500 \mathrm{AU}$ and $V_{0}=40 \mathrm{~km} \mathrm{~s}^{-1}$, one derives $t_{0} \approx 35 \mathrm{yr}$. Even assuming that the adopted values of $R_{0}$ and $V_{0}$ are wrong by $50 \%$, the maximum allowed value of $t_{0}$ is $\approx 80 \mathrm{yr}$.

In Appendix B. 1 it is shown that the pressure-driven shell can reproduce the observed values of radius $\left(R_{\mathrm{S}}=R_{0}\right)$ and velocity $\left(V_{\mathrm{s}}=V_{0}\right)$ only if the mechanical luminosity is below a threshold value, $L_{36} \leq L_{\mathrm{th}}$, where $L_{\mathrm{th}}$ is given by Eq. (B.8). Using the fiducial value $K_{0}=1$ for the thermal conductivity coefficient, and $R_{0}=500 \mathrm{AU}, V_{0}=40 \mathrm{~km} \mathrm{~s}^{-1}$, one derives $L_{\mathrm{th}} \approx 2.14$.

As discussed in Appendix B.2, when $L_{36} \geq L_{\mathrm{th}}$, wind-driven solutions reproducing the observed values of the shell radius and velocity, are obtained only in the momentum-driven phase. As for the pressure-driven case, for a given wind luminosity the solution requires a specific value of the ambient density. Using the fiducial value $K_{0}=1$, and $R_{0}=500 \mathrm{AU}, V_{0}=40 \mathrm{~km} \mathrm{~s}^{-1}$, for $2.14 \leq L_{36} \leq 10\left(2 \leq \dot{M}_{-6} \leq 8\right.$ assuming $\left.V_{2000}=1\right)$ the value of ambient density increases with the wind luminosity and varies in the range $139 \leq n_{5} \leq 250$. For $L_{36}=2.14$ and $n_{5}=139$, one obtains $t_{0}=t_{\mathrm{cr}}=35 \mathrm{yr}\left(R_{\mathrm{cr}}=500 \mathrm{AU}, V_{\mathrm{cr}}=40 \mathrm{~km} \mathrm{~s}^{-1}\right)$; increasing the value of the wind luminosity, while $t_{\mathrm{cr}}$ decreases rapidly (reaching a minimum value of $t_{\mathrm{cr}}=15 \mathrm{yr}$ for $L_{36}=10$ ), $t_{0}$ remains almost constant (at $L_{36}=10, t_{0}=30 \mathrm{yr}$ ). Appendix B.2 shows that one finds a value $t_{0} \approx 30 \mathrm{yr}$ also for very high values of wind luminosity $\left(L_{36} \gg 10\right)$ and ambient density $\left(n_{5} \geq 500\right)$.

Figure A.1 resumes the results of our calculations. It is reported the dependence of the shell age, $t_{0}$, and the $\mathrm{H}$ particle ambient density, $n_{5}$, on the mechanical wind luminosity, $L_{36}$, varying the wind velocity, $V_{2000}$, over a plausible range of values and considering that the measured shell parameters, $R_{0}$ and $V_{0}$, can be uncertain at most by $30 \%$. Note that the shell age is a constant function of the wind luminosity in the pressure-driven phase, and shows only a weak dependence in the momentum-driven phase. Note also that the $\mathrm{H}$ particle ambient density increases monotonically with the wind luminosity.

\section{Appendix B: Determination of the wind-driven solutions}

In the following, separately for the pressure and momentumdriven phases, we derive the relation between the mechanical wind luminosity and the ambient density that must be satisfied for the wind-driven shell to match the observed values of $R_{\mathrm{S}}=R_{0}$ and $V_{\mathrm{s}}=V_{0}$ at the same time.

\section{B.1. Pressure-driven phase}

By substituting $t / t_{\mathrm{cr}}$ from Eq. (A.4) into Eq. (A.5), one obtains:

$V_{\mathrm{s}} R_{\mathrm{s}}^{2 / 3}=V_{\mathrm{cr}} R_{\mathrm{cr}}^{2 / 3}$.

Requiring that $R_{\mathrm{S}}=R_{0}$ and $V_{\mathrm{S}}=V_{0}$, and expressing $R_{\mathrm{cr}}$ and $V_{\text {cr }}$ through Eqs. (A.2) and (A.3), one derives a relation between the ambient density and the wind mechanical luminosity that 


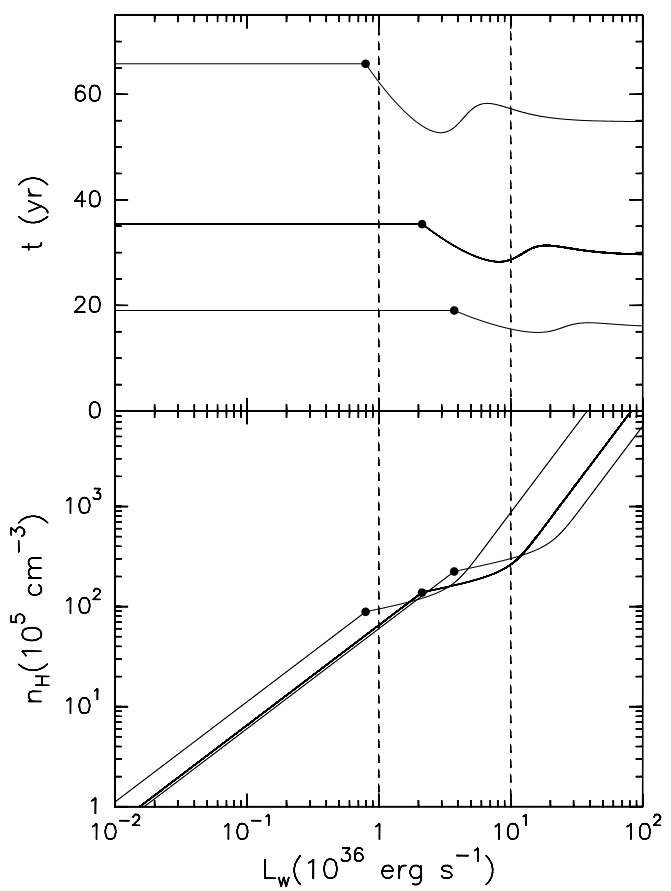

Fig. A.1. Upper and bottom panels show the dependence of the shell age and the $\mathrm{H}$ particle ambient density on the mechanical wind luminosity, respectively. In deriving these curves, we have always used the fiducial value $K_{0}=1$ for the thermal conductivity coefficient. In both panels, the thick line shows the result when the most likely value of wind velocity, $V_{2000}=1$, shell radius, $R_{0}=500 \mathrm{AU}$, and shell velocity, $V_{0}=40 \mathrm{~km} \mathrm{~s}^{-1}$, are used. The thin lines indicate the maximum expected excursion of the curves varying the previous parameters over a plausible range of values. In particular, in both panels, the upper thin line corresponds to the choice: $V_{2000}=0.75, R_{0}=650 \mathrm{AU}$ and $V_{0}=28 \mathrm{~km} \mathrm{~s}^{-1}$, while the lower thin line refers to the selection: $V_{2000}=1.25, R_{0}=350 \mathrm{AU}$ and $V_{0}=52 \mathrm{~km} \mathrm{~s}^{-1}$. The dot in each curve marks the luminosity threshold separating the pressure-driven from the momentum-driven solution. In both panels, the vertical dashed lines delimit the plausible range of variation of the mechanical wind luminosity of a ZAMS O9.5 star (Lamers \& Leitherer 1993).

must be satisfied for the pressure-driven shell to reproduce the observations:

$n_{5}=\left(\frac{V_{0}}{9.64 \mathrm{~km} \mathrm{~s}^{-1}}\right)^{-3}\left(\frac{R_{0}}{34034 \mathrm{AU}}\right)^{-2} L_{36}$.

With this relationship, the expressions for the critical time, radius and velocity can be rewritten as:

$$
\begin{aligned}
t_{\mathrm{cr}}= & \left(10^{4} \mathrm{yr}\right)\left(\frac{V_{0}}{9.64 \mathrm{~km} \mathrm{~s}^{-1}}\right)^{27 / 8} \\
& \times\left(\frac{R_{0}}{34034 \mathrm{AU}}\right)^{9 / 4} L_{36}^{-5 / 4} K_{0}^{-15 / 16} \\
R_{\mathrm{cr}}= & (34034 \mathrm{AU})\left(\frac{V_{0}}{9.64 \mathrm{~km} \mathrm{~s}^{-1}}\right)^{21 / 8} \\
& \times\left(\frac{R_{0}}{34034 \mathrm{AU}}\right)^{7 / 4} L_{36}^{-3 / 4} K_{0}^{-9 / 16} \\
V_{\mathrm{cr}}= & \left(9.64 \mathrm{~km} \mathrm{~s}^{-1}\right)\left(\frac{V_{0}}{9.64 \mathrm{~km} \mathrm{~s}^{-1}}\right)^{-3 / 4} \\
& \times\left(\frac{R_{0}}{34034 \mathrm{AU}}\right)^{-1 / 2} L_{36}^{1 / 2} K_{0}^{3 / 8} .
\end{aligned}
$$

Replacing the above expressions for $t_{\mathrm{cr}}$ and $R_{\mathrm{cr}}$ in Eq. (A.4), one finds that the expansion law of the shell radius does not depend any longer on the wind luminosity, $L_{36}$, and the thermal conductivity coefficient, $K_{0}$, provided Eq. (B.2) is satisfied:

$$
\begin{aligned}
R_{\mathrm{S}}= & (135.5 \mathrm{AU})\left(\frac{V_{0}}{9.64 \mathrm{~km} \mathrm{~s}^{-1}}\right)^{3 / 5} \\
& \times\left(\frac{R_{0}}{34034 \mathrm{AU}}\right)^{2 / 5}\left(\frac{t}{1 \mathrm{yr}}\right)^{3 / 5} .
\end{aligned}
$$

Requiring that $R_{\mathrm{s}}=R_{0}$ in Eq. (B.6), one derives the time $t_{0}$ at which the pressure-driven shell matches the shell parameters:

$t_{0}=\left(10^{4} \mathrm{yr}\right)\left(\frac{V_{0}}{9.64 \mathrm{~km} \mathrm{~s}^{-1}}\right)^{-1}\left(\frac{R_{0}}{34034 \mathrm{AU}}\right)$.

Using the observed values $R_{0}=500 \mathrm{AU}$ and $V_{0}=40 \mathrm{~km} \mathrm{~s}^{-1}$, one derives a shell age $t_{0}=35 \mathrm{yr}$.

From Eqs. (B.7) and (B.3), one can see that $t_{0} \geq t_{\mathrm{cr}}$ if the mechanical luminosity is above a threshold value given by:

$L_{\mathrm{th}}=\left(\frac{V_{0}}{9.64 \mathrm{~km} \mathrm{~s}^{-1}}\right)^{7 / 2}\left(\frac{R_{0}}{34034 \mathrm{AU}}\right) K_{0}^{-3 / 4}$.

Therefore for $L>L_{\text {th }}$ the pressure-driven solution is not valid and one has to consider the momentum-driven solution discussed in Appendix B.2. Using the fiducial value $K_{0}=1$ for the thermal conductivity coefficient, and $R_{0}=500 \mathrm{AU}, V_{0}=$ $40 \mathrm{~km} \mathrm{~s}^{-1}$, the upper limit for the wind luminosity is $L_{\mathrm{th}}=2.14$.

\section{B.2. Momentum-driven phase}

Requiring $R_{\mathrm{S}}=R_{0}$ and $V_{\mathrm{s}}=V_{0}$ for $t=t_{0}$, Eq. (A.6) and (A.7) can be rewritten as:

$$
\begin{aligned}
& t_{0}=t_{\mathrm{cr}}\left\{1+\frac{6}{5 C_{1}}\left[\sqrt{1+\frac{25}{36} C_{1}\left(\left(\frac{R_{0}}{R_{\mathrm{cr}}}\right)^{4}-1\right)}-1\right]\right\} \\
& \left(\frac{V_{0}}{V_{\mathrm{cr}}}\right)^{2}\left(\frac{R_{0}}{R_{\mathrm{cr}}}\right)^{6}=1+\frac{25}{36} C_{1}\left[\left(\frac{R_{0}}{R_{\mathrm{cr}}}\right)^{4}-1\right] .
\end{aligned}
$$

The latter is an equation relating $L_{36}$ to $n_{5}$ and represents the condition that must be satisfied for the momentum-driven shell to match the observed values of $R_{\mathrm{s}}=R_{0}$ and $V_{\mathrm{s}}=V_{0}$ at a given time.

For the following calculations, it is convenient to define the variable $f$ as:

$f^{19 / 4} \equiv\left(\frac{V_{0}}{V_{\mathrm{cr}}}\right)^{2}\left(\frac{R_{0}}{R_{\mathrm{cr}}}\right)^{6}$.

If Eq. (B.10) holds, by definition $f \geq 1$, because we deal with the momentum-driven phase for which $t_{0} \geq t_{\mathrm{cr}}$ and $R_{0} \geq R_{\mathrm{cr}}$. Replacing $R_{\text {cr }}$ and $V_{\text {cr }}$ in Eq. (B.11) using Eqs. (A.2) and (A.3), one may express $f$ as a function of the ambient density and the wind luminosity:

$$
\begin{aligned}
f= & \left(\frac{V_{0}}{9.64 \mathrm{~km} \mathrm{~s}^{-1}}\right)^{8 / 19} \\
& \times\left(\frac{R_{0}}{34034 \mathrm{AU}}\right)^{24 / 19} K_{0}^{21 / 38} n_{5} L_{36}^{-5 / 19} .
\end{aligned}
$$

By using the variable $f$, the terms appearing in the relation B.10 can be rewritten as:

$\left(\frac{V_{0}}{V_{\mathrm{cr}}}\right)^{2}\left(\frac{R_{0}}{R_{\mathrm{cr}}}\right)^{6}=f^{19 / 4}$ 


$$
\begin{aligned}
& \frac{25}{36} C_{1}\left(\frac{R_{0}}{R_{\mathrm{cr}}}\right)^{4}=1.51 \times 10^{-2}\left(\frac{V_{0}}{9.64 \mathrm{~km} \mathrm{~s}^{-1}}\right)^{-30 / 19} \\
& \times\left(\frac{R_{0}}{34034 \mathrm{AU}}\right)^{-14 / 19} K_{0}^{21 / 38} V_{2000}^{-4} L_{36}^{113 / 76} f^{15 / 4} \\
& \frac{25}{36} C_{1}= 1.51 \times 10^{-2}\left(\frac{V_{0}}{9.64 \mathrm{~km} \mathrm{~s}^{-1}}\right)^{-2 / 19} \\
& \times\left(\frac{R_{0}}{34034 \mathrm{AU}}\right)^{-6 / 19} K_{0}^{9 / 38} V_{2000}^{-4} L_{36}^{81 / 76} f^{1 / 4}
\end{aligned}
$$

and Eq. (B.10) becomes an equation for $f$ :

$$
f^{15 / 4}(f-G)=1-R f^{1 / 4}
$$

where we have used the notations:

$$
\begin{aligned}
G \equiv & 1.51 \times 10^{-2}\left(\frac{V_{0}}{9.64 \mathrm{~km} \mathrm{~s}^{-1}}\right)^{-30 / 19} \\
& \times\left(\frac{R_{0}}{34034 \mathrm{AU}}\right)^{-14 / 19} K_{0}^{21 / 38} V_{2000}^{-4} L_{36}^{113 / 76} \\
R \equiv & 1.51 \times 10^{-2}\left(\frac{V_{0}}{9.64 \mathrm{~km} \mathrm{~s}^{-1}}\right)^{-2 / 19} \\
& \times\left(\frac{R_{0}}{34034 \mathrm{AU}}\right)^{-6 / 19} K_{0}^{9 / 38} V_{2000}^{-4} L_{36}^{81 / 76}
\end{aligned}
$$

Using the fiducial value $K_{0}=1$ for the thermal conductivity coefficient, taking $R_{0}=500 \mathrm{AU}$ and $V_{0}=40 \mathrm{~km} \mathrm{~s}^{-1}$, when the wind luminosity and velocity vary over the assumed range of values, $R$ and $G$ fall inside the interval 0.01-3.4.

In Appendix $\mathrm{C}$ it is shown that for $f>1$, requiring also $L_{36} \geq L_{\text {th }}$, Eq. (B.16) has unique solution. Assuming $K_{0}=1$, $R_{0}=500 \mathrm{AU}$, and $V_{0}=40 \mathrm{~km} \mathrm{~s}^{-1}$, for values of wind luminosity $2.14 \leq L_{36} \leq 10$ and $V_{2000}=1$, the solution requires the value of $\mathrm{H}$ particle ambient density to vary in the range $139 \leq n_{5} \leq 250$.

We have evaluated the time $t_{0}$ at which the momentumdriven shell matches the observed values of radius and velocity. Using $K_{0}=1, R_{0}=500 \mathrm{AU}$, and $V_{0}=40 \mathrm{~km} \mathrm{~s}^{-1}$, for $L_{36}=L_{\mathrm{th}}=2.14, t_{0}=t_{\mathrm{cr}}=35$ yr. For $2.14 \leq L_{36} \leq 10$ (with $V_{2000}=1$ ), whereas $t_{\text {cr }}$ reduces to $15 \mathrm{yr}, t_{0}$ remains almost constant, varying in the range $35-30 \mathrm{yr}$. It is to be noted that, also for very high values of wind luminosities and mass loss rates, $t_{0}$ remains around the value of the pressure-driven shell solution (see Eq. (B.7)). This is demonstrated in the following.

From Eq. (B.9) and using Eqs. (B.10) and (B.13), one obtains:

$$
\begin{aligned}
t_{0} & =t_{\mathrm{cr}}\left[1+\frac{6}{5 C_{1}}\left(f^{19 / 8}-1\right)\right] \\
& =t_{\mathrm{cr}}\left[1+\frac{5}{6 R f^{1 / 4}}\left(f^{19 / 8}-1\right)\right]
\end{aligned}
$$

where in the last equality we have replaced $C_{1}=36 / 25 R f^{1 / 4}$, using Eqs. (B.15) and (B.18).

Assuming that $R_{0}$ and $V_{0}$ can vary at most by $50 \%$ around the observed values (500 AU and $40 \mathrm{~km} \mathrm{~s}^{-1}$, respectively), with $0.75 \leq V_{2000} \leq 1.25$ and $K_{0}=1$, from Eqs. (B.17) and (B.18) one can see that $G>R>1$ if $L_{36} \gg 10$. As shown in Appendix $\mathrm{C}$, for high values of $R$ and $G, f \approx G$, so that it must also be $f>1$ and $f^{19 / 8} \gg 1$. If $f \approx G>R>1$ and $f^{19 / 8} \gg 1$, then, from Eq. (B.19), $t_{0} \gg t_{\mathrm{cr}}$ and one can write:

$$
\begin{aligned}
t_{0} \approx & t_{\mathrm{cr}} \frac{5}{6 R f^{1 / 4}} f^{19 / 8} \approx\left(10^{4} \mathrm{yr}\right)\left(\frac{V_{0}}{9.64 \mathrm{~km} \mathrm{~s}^{-1}}\right)^{9 / 19} \\
& \times\left(\frac{R_{0}}{34034 \mathrm{AU}}\right)^{27 / 19} K_{0}^{-6 / 19} L_{36}^{-8 / 19} f^{-9 / 8} \frac{5}{6 R} f^{17 / 8}
\end{aligned}
$$

where we have replaced $t_{\text {cr }}$ with the Eq. (A.1), using Eq. (B.12) to express the ambient density $n_{5}$ as a function of $f$.

Again taking into account that for high values of wind luminosity $R>1, G>1$ and $f \approx G$, one finds:

$$
\begin{aligned}
t_{0} \approx & \frac{5}{6}\left(10^{4} \mathrm{yr}\right)\left(\frac{V_{0}}{9.64 \mathrm{~km} \mathrm{~s}^{-1}}\right)^{9 / 19} \\
& \times\left(\frac{R_{0}}{34034 \mathrm{AU}}\right)^{27 / 19} K_{0}^{-6 / 19} L_{36}^{-8 / 19} \frac{G}{R} \\
= & \frac{5}{6}\left(10^{4} \mathrm{yr}\right)\left(\frac{V_{0}}{9.64 \mathrm{~km} \mathrm{~s}^{-1}}\right)^{-1}\left(\frac{R_{0}}{34034 \mathrm{AU}}\right)
\end{aligned}
$$

where we have used the expression:

$\frac{G}{R}=\left(\frac{V_{0}}{9.64 \mathrm{~km} \mathrm{~s}^{-1}}\right)^{-28 / 19}\left(\frac{R_{0}}{34034 \mathrm{AU}}\right)^{-8 / 19} K_{0}^{6 / 19} L_{36}^{8 / 19}$

obtained from Eqs. (B.17) and (B.18).

Equation (B.21) demonstrates that the value of $t_{0}$ for high wind luminosities is very close to the pressure driven solution, given in Eq. (B.7).

\section{Appendix C: Existence and uniqueness of the momentum-driven solution}

The purpose of this appendix is to establish under which conditions Eq. (B.16) has one and only one solution. Let us consider separately the left and right handsides of Eq. (B.16):

$H(f) \equiv f^{15 / 4}(f-G)$

$K(f) \equiv 1-R f^{1 / 4}$

The function $H(f)$ has a minimum for $f=f_{\mathrm{m}}=15 / 19 G$, while $K(f)$ is always decreasing with $f$. When $G<19 / 15$, for $f \geq 1, H(f)$ monotonically increases with $f$, and no solutions of Eq. (B.16) exist unless $H(1) \leq K(1)$, or equivalently $G \geq R$. That brings to the condition $L_{36} \geq L_{\mathrm{th}}$ (see Eq. (B.8)), which, as also noted in Appendix B.1, is the threshold in wind luminosity that separates the pressure-driven from the momentum-driven solutions. If $G<19 / 15$ (with $G \geq R$ ), the two functions $H(f)$ and $K(f)$ are respectively monotonically increasing and decreasing with $f$, which assures the existence of a single solution for Eq. (B.16). On the other hand, if $G \geq 19 / 15$ one can show that for $L_{36} \geq L_{\mathrm{th}}, K(f) \geq H(f)$ for $1 \leq f \leq f_{\mathrm{m}}$, and since for $f>f_{\mathrm{m}}$ again $H(f)$ and $K(f)$ are respectively monotonically increasing and decreasing with $f$, the existence of a single solution is assured also in this case. One finds that the value of the solution $f$ increases with $R$ and $G$ (i.e. increases with $L_{\mathrm{w}}$ and decreases with $V_{\mathrm{w}}$ ). Using the fiducial value $K_{0}=1$, and $R_{0}=500 \mathrm{AU}$, $V_{0}=40 \mathrm{~km} \mathrm{~s}^{-1}$, one derives that the solution of Eq. (B.16) satisfies the following constraints:

1. if $R \leq 1$ and $G \leq 1$ :

$1 \leq f \leq 1.22$

2. if $R \leq 1$ and $G \geq 1$ (i.e. $0.5 \leq R \leq 1$ and $1 \leq G \leq 2$ ):

$$
|f-G| \leq 1-R
$$

3. if $R \geq 1$ and $G \geq 1$ :

$$
\frac{15}{19} G \leq f \leq G
$$

